TRANSACTIONS OF THE

AMERICAN MATHEMATICAL SOCIETY

Volume 349, Number 2, February 1997, Pages 713-746

S 0002-9947(97)01853-9

\title{
A LIE THEORETIC GALOIS THEORY FOR THE SPECTRAL CURVES OF AN INTEGRABLE SYSTEM. II
}

\author{
ANDREW MCDANIEL AND LAWRENCE SMOLINSKY
}

\begin{abstract}
In the study of integrable systems of ODE's arising from a Lax pair with a parameter, the constants of the motion occur as spectral curves. Many of these systems are algebraically completely integrable in that they linearize on the Jacobian of a spectral curve. In an earlier paper the authors gave a classification of the spectral curves in terms of the Weyl group and arranged the spectral curves in a hierarchy. This paper examines the Jacobians of the spectral curves, again exploiting the Weyl group action. A hierarchy of Jacobians will give a basis of comparison for flows from various representations. A construction of V. Kanev is generalized and the Jacobians of the spectral curves are analyzed for abelian subvarieties. Prym-Tjurin varieties are studied using the group ring of the Weyl group $W$ and the Hecke algebra of double cosets of a parabolic subgroup of $W$. For each algebra a subtorus is identified that agrees with Kanev's Prym-Tjurin variety when his is defined. The example of the periodic Toda lattice is pursued.
\end{abstract}

\section{INTRODUCTION}

This paper continues the work begun in part one. Part one gave a classification of spectral curves in terms of covering space theory and the action of Weyl group. This paper examines the Jacobians of the spectral curves, again exploiting the Weyl group action. The spectral curves studied arise from completely integrable systems described as a Lax pair with a parameter. The system is expressed in the form $\frac{d A}{d t}=[A, B]$ where

(1) $A$ and $B$ lie in a Lie algebra $\mathfrak{g}$,

(2) $A$ and $B$ are functions of time $t$ and rational functions of a parameter $s$ where $s$ is a coordinate on an algebraic curve $P$.

For each representation $\rho$ of $\mathfrak{g}$, the characteristic polynomial of $\rho(A)$ defines a curve by the equation $0=\operatorname{det}(\rho A(s, t)-z)$. It is a consequence of the Lax form of the differential equation for $A$ that $\{(s, z) \mid 0=\operatorname{det}(\rho A(s, t)-z)\}$ is independent of time for any representation $\rho$. These curves of eigenvalues are in general reducible and the irreducible components of these various curves and certain combinations of components (when normalized) are called spectral curves. Part of the interest of this description is that the flow of the system can often be described as occurring on the Jacobian of a spectral curve; i.e., a Hamiltonian flow torus can be produced in the

Received by the editors February 22, 1995.

1991 Mathematics Subject Classification. Primary 58F05, 58F07.

The second author was partially supported by a Louisiana Education Quality Support Fund grant LEQSF(90-93)-RD-A-10. 
Jacobian. The process was first described by van Moerbeke and Mumford [vMM] for the case of the periodic Toda lattice and some generalizations. Although the spectral curve (of eigenvalues) does not change with time the associated eigenvectors do. At each time the eigenvectors determine a bundle over the spectral curve and give a point in the moduli space of line bundles. Translating to the Jacobian is the desired flow.

In [AvM2] a Prym variety contained in the Jacobian of the spectral curve of the representation with highest weight $\lambda_{1}$ was produced for the algebras $B_{n}, C_{n}, D_{n}$ and $G_{2}$. Adler and van Moerbeke showed that the Toda lattice flows linearized on these subtori. The most general results in this direction are by Vassil Kanev $[\mathrm{K}]$ when $P$ is the Riemann sphere. He showed that in the case of the algebras $A_{n}, D_{n}$, $E_{6}, E_{7}$ and $E_{8}$ there is a Prym-Tjurin variety in the Jacobian and that for spectral curves of minuscule weights in $A_{n}, D_{n}, E_{6}$ and $E_{7}$ the Prym-Tjurin has a principal polarization. Prym-Tjurin varieties are abelian varieties which are generalizations of Prym varieties. Under technical conditions (see 8.4 of $[\mathrm{K}]$ ) he showed the flow is restricted to the Prym-Tjurin.

In this paper we construct the hierarchy of Jacobians of spectral curves. This hierarchy will serve as a framework for a comparison of the flows from various representations, which will be done in a future paper. The flows can be compared on the Jacobian of the curve at the top of the covering space hierarchy. The map up from any smaller Jacobian has at most $\mathbf{Z}_{2}^{k}$ as a kernel. Hence the loss of information is two torsion while Adler and van Moerbeke determined it was finite. The covering space theory leads to decompositions of the Jacobians, permitting a generalization of Kanev's construction and an extension of his results. The PrymTjurin varieties are studied using the group ring of the Weyl group $W$ and the Hecke algebra of double cosets of a parabolic subgroup of $W$. For each algebra a subtorus is identified. This subtorus agrees with Kanev's Prym-Tjurin variety when his is defined. In the case when the algebra is $A_{n}$ and the parameter space is the Riemann sphere the subtorus is isogenous to a Jacobian. In the case of the algebras $B_{n}, C_{n}$ and $D_{n}$ this subtorus is isogenous to a Prym variety. For the exceptional algebras the subtorus is isogenous to a Prym-Tjurin and we expect it can fail to be isogenous to a Prym. The example of the periodic Toda lattice is pursued. Flows are not pursued here. However Kanev's condition does not apply to well-known systems. In [MS1] it is shown that the $G_{2}$ periodic Toda flow is in the 2-dimensional Prym-Tjurin. This result identifies the 2-dimensional Hamiltonian torus in the 3-dimensional Prym variety produced by Adler and van Moerbeke [AvM2].

After discussion of Notation and assumptions in section 1 a Hierarchy of Jacobians related to an initial condition is introduced in section 2. These Jacobians are derived from the hierarchy of spectral curves. In section 3 Hecke rings are shown to act as correspondences on the curves. In section 4, Endomorphisms of the Jacobi variety and Prym-Tjurin varieties, the subtorus is produced. Section 5 produces a Lefschetz type formula for the dimension of the PrymTjurin. In section 6, Pryms and Jacobians, the question "when is the subtorus actually isogenous to a Prym or Jacobian" is explored. In the final section, section 7, the Periodic Toda lattice is studied. The Prym variety of the $\lambda_{1}$ flow is shown to be determined up to isomorphism rather than isogeny for $\mathfrak{g}$ of type $A, B, C$, $D$, and $G_{2}$. The dimension formula is then applied. The paper concludes with a 
detailed analysis of the Jacobian of the $s l(4, \mathbf{C})$ Toda master curve, a continuation of the example from Paper I.

\section{Notation AND ASSUMPtions}

We first establish our notation. $G$ is a simply-connected complex semi-simple Lie group with Lie algebra $\mathfrak{g}$. When specifying simple Lie algebras by their type, we adhere to the standard policy that $n \geq 2$ for types $B_{n}$ and $C_{n}$ and $n \geq 4$ for type $D_{n} . H$ is a Cartan subgroup (a maximal torus as $G$ is semi-simple) with Lie algebra $\mathfrak{h}$. $W$ denotes the Weyl group, $W=N_{H} / C_{H}$, the normalizer of $H$ modulo the centralizer of $H$. Since $G$ is semi-simple $C_{H}=H$. A basis of simple roots $\Delta=\left\{\alpha_{1}, \ldots, \alpha_{r}\right\}$ has been chosen. Let $B=B^{+}$and $B^{-}$be the pair of opposite Borel subgroups of $G$, whose intersection is $H$, determined by the choice of a basis of simple roots. Recall that the real span of $\Delta=\left\{\alpha_{1}, \ldots, \alpha_{r}\right\}$ is a Euclidean subspace of the dual vector space $\mathfrak{h}^{*}$ of the Cartan subalgebra $\mathfrak{h}$ with inner product $\langle$,$\rangle given by the dual of the Killing form. The weight lattice is$ $\left\{\lambda \in \mathfrak{h}^{*} \mid \frac{2\langle\lambda, \alpha\rangle}{\langle\alpha . \alpha\rangle} \in \mathbf{Z} \forall \alpha \in \Delta\right\}$. The Weyl group $W$ acts on the weight lattice by the coadjoint action $(n H \cdot \lambda)(X)=\lambda\left(A d_{n} X\right)$ for $X \in \mathfrak{h}$. The stabilizer in $W$ of a weight $\lambda$ will be denoted $S_{\lambda}$. We call $S_{\lambda}$ a parabolic subgroup of the Weyl group.

Occasionally a sum or union will run through a set of coset or double coset representatives. This will be written as follows. If $B \subset A$ and $C \subset A$ are subgroups we write $x: A / B, x: B \backslash A$, and $x: B \backslash A / C$ for $x$ runs through representatives of left, right and double cosets.

Let the subscript ${ }_{*}$ refer to the regular elements in an algebra. Let $\pi: G / H \times$ $\mathfrak{h}_{*} \rightarrow \mathfrak{g}_{*}$ be given by $\pi(g H, h)=A d_{g} h$. This map is a regular cover with group of covering translations $W$. The action is given by $(n H) \cdot(g H, h)=\left(g n^{-1} H, A d_{n} h\right)$ for $n \in N_{H}$. Let $A$ be a morphism from an irreducible algebraic curve $P$ to a Lie algebra $\mathfrak{g}$ with $\operatorname{im}(A) \cap \mathfrak{g}_{*} \neq \emptyset$. Then $A^{-1}\left(\mathfrak{g}_{*}\right)$ is a Zariski open set in $P$ (i.e., $P \backslash$ finite set) denoted $P_{*}$. We call $P$ the parameter space as it is the domain for the spectral parameter occurring in the Lax equation. Let $\epsilon: \pi_{1}\left(\mathfrak{g}_{*}\right) \rightarrow W$ be the map determined by the $W$ cover. Let $\mu: \pi_{1}\left(P_{*}\right) \rightarrow W$ be the composition $\epsilon \circ A_{*}$ where $A_{*}$ is the map on fundamental groups induced by the map $A: P_{*} \rightarrow \mathfrak{g}_{*}$.

If $\rho$ is a representation then the curve $\{(s, z) \in P \times \mathbf{C} \mid \operatorname{det}(\rho A(s)-z)=0\}$ is in general reducible and decomposes via the dominant weights. These pieces are independent of the representation in that they depend only on the weights. If $m_{\lambda}$ is the multiplicity of the weight $\lambda$ in $\rho$ then

$$
\operatorname{det}(\rho A(s)-z)=\prod_{\lambda}\left(p_{\lambda}(s, z)\right)^{m_{\lambda}}
$$

where $\lambda$ runs through the dominant weights (see [MS]).

Let $Y_{\lambda}$ denote the normalization of the variety defined by $p_{\lambda}(s, z)=0$. This is the notion of spectral curve used by $\mathrm{V}$. Kanev. These curves themselves may decompose. We will reserve the notation of $Y$ for these possibly disconnected spectral curves and the notation $X$ for irreducible components of the disconnected spectral curves. In Paper I the irreducible components were referred to as the spectral curves and a complete classification was given. In this paper we follow in the direction of Kanev and study the disconnected spectral curves, $Y_{\lambda}$. Let $Y_{\lambda} *$ be the inverse image of $P_{*}$ in $Y_{\lambda}$. 
Let the image of the map $\mu: \pi_{1}\left(P_{*}\right) \rightarrow W$ be $M \subset W$. Let $Y_{*}$ be the pullback of

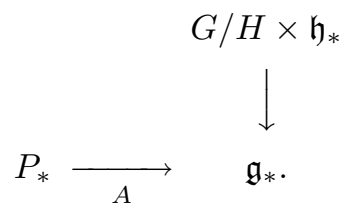

So $Y_{*}$ is a principal $W$ bundle. Let $X_{*}$ be an irreducible component of $Y_{*}$. Then $X_{*}$ is a principal $M$ bundle and $Y_{*}=\bigcup_{w: W / M} w X_{*}$. See [MS], pages 137 and 138 for details. From Theorem 13, [MS] and its proof, we have:

Proposition 1. If $\lambda$ is a weight then $Y_{\lambda *} \cong Y_{*} / S_{\lambda}$. Moreover $Y_{\lambda *}$ is the pullback of

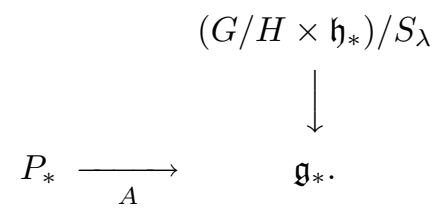

Furthermore $Y_{\lambda *} \cong \bigcup_{w: S_{\lambda} \backslash W / M} X_{*} /\left(M \cap w^{-1} S_{\lambda} w\right) \cong \bigcup_{w: S_{\lambda} \backslash W / M} X_{*} /\left(M \cap S_{w^{-1} \cdot \lambda}\right)$.

We use $Y_{\lambda}$ and $Y / S_{\lambda}$ interchangeably.

Note that if $S_{\lambda}$ and $S_{\gamma}$ are conjugate subgroups of $W$ then $Y_{\lambda} \cong Y_{\gamma}$ not only as varieties but also as quotients of $W$-bundles. It is possible for $Y_{\lambda}$ and $Y_{\gamma}$ to be isomorphic as varieties even if $S_{\lambda}$ and $S_{\gamma}$ are not conjugate. For example, take $P$ the Riemann sphere, $\mathfrak{g}=A_{6}, M$ trivial, $S_{\lambda}=\Sigma_{3} \oplus \Sigma_{2} \oplus \Sigma_{2}$ and, $S_{\gamma}=\Sigma_{4} \oplus \Sigma_{1} \oplus \Sigma_{1} \oplus \Sigma_{1}$; then $Y_{\lambda} \cong Y_{\gamma}$ is a union of 210 Riemann spheres. Our analysis will rely on the $W$ action so we will identify $Y_{\lambda}$ and $Y_{\gamma}$ whenever $S_{\lambda}$ and $S_{\gamma}$ are conjugate subgroups. Note that if $\mu$ is onto then $Y_{\lambda}$ is connected for all $\lambda$. If $S_{\lambda}=\{e\}$ then $Y_{\lambda} \cong Y$ and each component is isomorphic to $X$. We call both of these curves $Y$ and $X$ the master spectral curves.

We will need to consider ramified covers and disconnected ramified covers of curves which may be reducible. All will be finite-to-one maps. In a disconnected ramified cover each map between components is a ramified cover and all fibers over nonramification points have the same cardinality. We will also need branched $K$ bundles with $K$ a finite group. Off the inverse image of the ramification points, the total space is a $K$ bundle. Each component of the total space is a ramified cover of its image and the total space modulo the $K$ action is the base space. We will call an automorphism in $K$ a deck transformation since a branched $K$ bundle is a disconnected regular branched cover.

Let Jac $R$ be the Jacobian of a curve $R$. If $R$ is reducible then Jac $R$ denotes $P i c^{(0, \cdots, 0)} R$. Suppose that $\pi: R_{1} \rightarrow R_{2}$ is a possibly disconnected ramified cover of curves. Then $\pi^{-1}: R_{2} \rightarrow R_{1}$ will be the inverse correspondence obtained by pulling back a point with its multiplicities. This is sometimes called the norm map. There are induced maps on divisors and so on the Jacobians which we write $\pi: \operatorname{Jac} R_{1} \rightarrow \operatorname{Jac} R_{2}$ and $\pi^{*}: \operatorname{Jac} R_{2} \rightarrow \operatorname{Jac} R_{1}$. We use $\pi^{*}$ for the map induced by $\pi^{-1}$. It is the pullback of bundles. We allow $\pi$ to denote both the forward map on bundles as well as the original map or correspondence on curves. Note that $\pi \circ \pi^{*}: \mathrm{Jac} R_{2} \rightarrow$ Jac $R_{2}$ is multiplication by the degree of the cover again assuming the degree is the same for each component of $R_{2}$. 


\section{HIERARCHY OF JACOBIANS}

The spectral curves are organized into a hierarchy of ramified covering spaces over a parameter space $P$. If $S_{\gamma} \subset S_{\lambda}$ then there is a ramified covering projection, $\pi_{\lambda \gamma}: Y_{\gamma} \rightarrow Y_{\lambda}$ such that

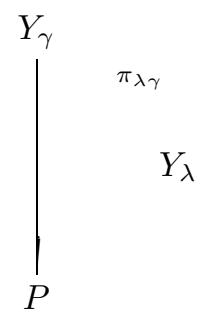

is a commutative diagram of ramified covers [MS]. Note that $Y_{0}=P$. We write $\pi_{\lambda}$ for the covering projection $\pi_{\lambda}: Y \rightarrow Y_{\lambda}$. When dealing with loops and fundamental groups, we shall assume that basepoints have been chosen for the various spectral curves such that the projection maps are basepoint preserving. The basepoint for $P$ is taken to be in $P_{*}$. The following theorem is an immediate consequence of the fact that $\pi_{\eta \gamma}=\pi_{\eta \lambda} \circ \pi_{\lambda \gamma}$.

Theorem 2. The hierarchy of spectral curves induces a hierarchy of Jacobians; i.e., if $S_{\gamma} \subset S_{\lambda}$ then $\pi_{\lambda \gamma}^{*}: \operatorname{Jac} Y_{\lambda} \rightarrow \operatorname{Jac} Y_{\gamma}$ and if $S_{\gamma} \subset S_{\lambda} \subset S_{\eta}$ then $\pi_{\eta \gamma}^{*}=$ $\pi_{\lambda \gamma}^{*} \circ \pi_{\eta \lambda}^{*}$

We now examine the kernel of $\pi_{\lambda \gamma}^{*}$. The following fact concerning Riemann surfaces is used (see $[\mathrm{A}]$, Lemma 2 which is stated without proof or [BL], pages 28 and 29 for a proof). Suppose that $R_{1} \rightarrow R$ is a ramified cover of Riemann surfaces and that $R_{U A} \rightarrow R$ is the maximal unramified abelian cover that makes the following diagram commute.

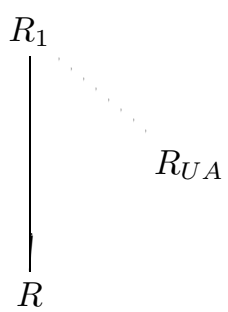

Call the abelian group of covering translations $A$. Then $\operatorname{ker}\left(\operatorname{Jac} R \rightarrow \operatorname{Jac} R_{1}\right)=A$.

Proposition 3. Jac $Y_{\lambda}$ is isogenous to im $\left(\pi_{\lambda \gamma}^{*}\right)$ under the homomorphism $\pi_{\lambda \gamma}^{*}$. If $\mu$ maps onto $W$ then $S_{\lambda}$ has a normal subgroup $N$ that contains $S_{\gamma}$ such that $S_{\lambda} / N \cong \operatorname{ker}\left(\pi_{\lambda \gamma}^{*}\right)$. Furthermore $S_{\lambda} / N$ is the group of covering translations of the maximal unramified abelian cover of $Y_{\lambda}$ which is a subcover of $Y_{\gamma}$.

Proof. Since $\pi_{\lambda \gamma} \circ \pi_{\lambda \gamma}^{*}: \operatorname{Jac} Y_{\lambda} \rightarrow \operatorname{Jac} Y_{\lambda}$ is multiplication by $\left|S_{\lambda}\right| /\left|S_{\gamma}\right|, \pi_{\lambda \gamma}^{*}$ is an isogeny onto its image. By Lemma 2 of $[\mathrm{A}]$, the kernel of $\pi_{\lambda \gamma}^{*}$ is a finite abelian group $A$ such that there is an unramified abelian cover $c: Y_{U A} \rightarrow Y_{\lambda}$ through which 
$\pi_{\lambda \gamma}$ factors, i.e.,

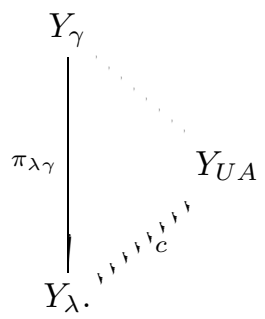

We have that $\pi_{1}\left(Y_{*}\right) \subset \pi_{1}\left(Y_{\gamma *}\right) \subset \pi_{1}\left(Y_{U A *}\right) \subset \pi_{1}\left(Y_{\lambda *}\right)$. Let $\pi_{1}\left(Y_{U A *}\right) / \pi_{1}\left(Y_{*}\right)=N$. Then $N$ is normal in $S_{\lambda}=\pi_{1}\left(Y_{\lambda *}\right) / \pi_{1}\left(Y_{*}\right)$ since $Y_{U A *}$ is a regular cover of $Y_{\lambda *}$. Also $\pi_{1}\left(Y_{\gamma^{*}}\right) / \pi_{1}\left(Y_{*}\right)=S_{\gamma}$ so $S_{\gamma} \subset N \triangleleft S_{\lambda}$. The group of covering translations is $S_{\lambda} / N$ and so is abelian.

Proposition 3 puts restrictions on the kernel of $\pi_{\lambda \gamma}^{*}$.

Proposition 4. If $\mu$ maps onto $W$ then $\operatorname{ker}\left(\pi_{\lambda \gamma}^{*}\right)$ is a sum of $\mathbf{Z}_{2}$ 's.

Proof. The kernel of the isogeny is an abelian quotient of a parabolic subgroup of $W$. The lemma below shows that the only such quotients are a direct sum of copies of $\mathbf{Z}_{2}$.

Lemma 5. If $W(\mathfrak{g})$ is the Weyl group of $\mathfrak{g}$ and $S \subset W(\mathfrak{g})$ is any proper parabolic subgroup then the abelianization of $S$ is a direct sum of $\mathbf{Z}_{2}$ 's.

Proof. Any proper parabolic subgroup of a Weyl group is the Weyl group of a proper subdiagram. These are direct sums of groups with summands Weyl groups of type $A_{n}, B_{n}, C_{n}, D_{n}, E_{6}$, or $E_{7}$. We need only check that the abelianization of any of these summands is either $\mathbf{Z}_{2}$ or $\mathbf{Z}_{2} \times \mathbf{Z}_{2}$. The abelianization of $S$ is $S$ modulo its commutator. For the Weyl groups $W\left(A_{n}\right), W\left(D_{n}\right)$ for $n>3, W\left(E_{6}\right)$ and $W\left(E_{7}\right)$ the commutator is an index 2 subgroup, the rotation subgroup ([H1], pages 44-45). So their abelianization is $\mathbf{Z}_{2}$. Also $W\left(B_{n}\right)$ and $W\left(C_{n}\right)$ are isomorphic to $\mathbf{Z}_{2} \times W\left(D_{n}\right)$. So their abelianization is $\mathbf{Z}_{2} \times \mathbf{Z}_{2}$.

More can be shown for specific systems by more careful analysis. In the section the Periodic Toda lattice it is shown that the kernel of $\pi_{\lambda \gamma}^{*}$ is trivial.

Let $\lambda$ be a dominant weight and $\rho$ the representation with highest weight $\lambda$. Define $v: Y_{\lambda *} \rightarrow \mathbf{C} P^{N-1}$ by the equation $\rho(A(s)) v(s, z)=z v(s, z)$. The map $v$ has a unique completion to $v: Y_{\lambda} \rightarrow \mathbf{C} P^{N-1}$ (see [G]) and is called the eigenvector map. For an algebraically completely integrable system the flow is realized as $v_{t}^{*}\left(\mathcal{O}_{\mathbf{C} P^{N-1}}(1)\right) \otimes v_{0}^{*}\left(\mathcal{O}_{\mathbf{C} P^{N-1}}(-1)\right)$ where $\mathcal{O}_{\mathbf{C} P^{N-1}}(1)$ is the line bundle over $\mathbf{C} P^{N-1}$ with divisor a hyperplane and $\mathcal{O}_{\mathbf{C} P^{N-1}}(-1)$ is its dual. This is a flow in $\operatorname{Jac} Y_{\lambda}$. However the flow can be lifted to Jac $Y_{\gamma}$ for any $\gamma$ such that $S_{\gamma} \subset S_{\lambda}$ via the map $\pi_{\lambda \gamma}^{*}$.

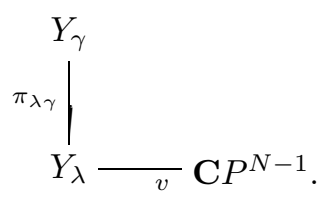

Note that all the flows live on the Jacobian of the master spectral curve via the $\operatorname{maps} \pi_{\lambda}^{*}$. 


\section{Correspondences}

This section describes rings of correspondences on the master curve $Y$ and on its quotient spectral curves, $Y_{\lambda}$. For the master curve this ring of correspondences is isomorphic to the integral group ring $\mathbf{Z}[W]$ of the Weyl group. For a quotient curve, $Y_{\lambda}$, this ring of correspondences is isomorphic to the Hecke ring $H\left(S_{\lambda}, W\right)$ of double cosets of $S_{\lambda}$ in $W$.

Let $\pi: Y \rightarrow P$ be the projection from the master curve to the parameter space. Each element of $W$ acts on $Y$ since $Y$ is a branched principal $W$ bundle and so may be regarded as a correspondence from $Y$ to $Y$. Forming integral linear combinations gives the following.

Proposition 6. $\mathbf{Z}[W]$ acts as a set of correspondences of $Y$.

We now turn to the correspondences on the quotient spectral curves. There is no $W$ action on $Y / S_{\gamma}$ as covering translations to extend to a $\mathbf{Z}[W]$ action of correspondences. There is an algebra of correspondences isomorphic to the Hecke ring $H\left(S_{\gamma}, W\right)$.

The description of the Hecke ring follows [Kr]. For $S$ a subgroup of $W$ the elements of $H(S, W)$ are the formal finite linear combinations of the double cosets $S w S$. So an element of $H(S, W)$ has the form $a=\sum_{g: S \backslash W / S} a(S g S) S g S$ where $a(S g S) \in \mathbf{Z}$ and $g$ runs through double coset representatives. As $\mathbf{Z}$ modules there is a natural identification of $H(S, W)$ with $\mathbf{Z}[S \backslash W]^{S}$, the module of linear combinations of right cosets $S w$ which are invariant under the right action of $S$. Specifically, $\sum_{g: S \backslash W} a(S g) S g$ is in $\mathbf{Z}[S \backslash W]^{S}$ if and only if it equals $\sum_{g: S \backslash W} a(S g) S g s$ for all $s \in S$. An element $\sum_{g: S \backslash W / S} a(S g S) S g S \in H(S, W)$ is identified with $\sum_{g: S \backslash W} a(S g S) S g \in \mathbf{Z}[S \backslash W]^{S}$; i.e. each double coset is a union of right cosets and each right coset is assigned the coefficient of the double coset it lies in. The multiplication in $H(S, W) \cong \mathbf{Z}[S \backslash W]^{S}$ is given by the map $\mathbf{Z}[S \backslash W]^{S} \times \mathbf{Z}[S \backslash W]^{S} \rightarrow$ $\mathbf{Z}[S \backslash W]^{S}$ which is the linear extension of $(S g)(S h)=S g h$. This is the multiplication of the quotient group when $S$ is a normal subgroup. But when $S$ is not normal this multiplication is not well-defined on right cosets. However it is well-defined when restricted to linear combinations of right cosets which are invariant under the right action of $S$.

Proposition 7. There is a monomorphism $\Phi$ from the Hecke algebra $H\left(S_{\lambda}, W\right)$ to the algebra of correspondences on $Y / S_{\lambda}$, as follows. For $x_{\lambda} \in Y / S_{\lambda}$, let $x$ be any preimage under the projection $\pi_{\lambda}: Y \rightarrow Y / S_{\lambda}$. Similarly for $a \in \mathbf{Z}\left[S_{\lambda} \backslash W\right]$ let $\bar{a}$ be any preimage of a under the projection map $\mathbf{Z}[W] \rightarrow \mathbf{Z}\left[S_{\lambda} \backslash W\right]$. Then $\Phi$ assigns to $a \in \mathbf{Z}\left[S_{\lambda} \backslash W\right]^{S_{\lambda}}$ the correspondence $C_{a}$ on $Y / S_{\lambda}$ defined by linear extension of

$$
C_{a}\left(x_{\lambda}\right)=\pi_{\lambda}(\bar{a} \cdot x)=\frac{1}{\left|S_{\lambda}\right|} \pi_{\lambda}\left(\bar{a} \cdot \pi_{\lambda}^{-1}\left(x_{\lambda}\right)\right) .
$$

Proof. First we check that $\pi_{\lambda}(\bar{a} \cdot x)$ is independent of the choice of $\bar{a}$ and of $x$ so that $C_{a}$ is well-defined. If $a=\sum_{w: S_{\lambda} \backslash W} a\left(S_{\lambda} w S_{\lambda}\right) S_{\lambda} w$, then

$$
\bar{a}=\sum_{w: S_{\lambda} \backslash W} a\left(S_{\lambda} w S_{\lambda}\right) s_{w} w
$$

where the choices of $s_{w} \in S_{\lambda}$ are arbitrary. Since $\pi_{\lambda}(s \cdot y)=\pi_{\lambda}(y)$ the choices of $s_{w}$ do not affect $\pi_{\lambda}(\bar{a} \cdot x)$. Similarly an alternative choice for $x$ has the form $s \cdot x$ for some $s \in S_{\lambda}$, but since $a=a s$ for any $s \in S_{\lambda}$, the value of $\pi_{\lambda}(\bar{a} \cdot x)$ is independent of 
$s$. Hence $C_{a}\left(x_{\lambda}\right)=\pi_{\lambda}(\bar{a} \cdot x)$ is well-defined. The second equality is just averaging over the inverse images of $x_{\lambda}$.

Secondly we check that $C_{a b}=C_{a} C_{b}$. Since $C_{a} C_{b}\left(x_{\lambda}\right)=\pi_{\lambda}(\bar{a} \cdot(\bar{b} \cdot x))=\pi_{\lambda}((\bar{a} \bar{b}) \cdot x)$, we need merely check that if $\bar{a}$ and $\bar{b}$ represent $a$ and $b$, then $\bar{a} \bar{b}$ represents $a b$. This follows from the definition of multiplication in the Hecke algebra.

\section{Endomorphisms of the Jacobi variety And Prym-TJurin varieties}

In this section Prym-Tjurin varieties within the Jacobi varieties of the spectral curves are defined. The various Prym-Tjurin varieties for a hierarchy of spectral curves are shown to be isogenous.

Every correspondence $C$ of a curve $R$ with itself extends linearly to a map from the divisors on $R$ to the divisors on $R$. Moreover, this map sends principal divisors to principal divisors, so respects linear equivalence. Hence, viewing the Jacobi variety of $R$ as the linear equivalence classes of degree zero divisors ( i.e. as $\operatorname{Pic}^{0} R$ ), a correspondence $C$ induces an endomorphism $C: \operatorname{Jac} R \rightarrow \operatorname{Jac} R$. The differential of this map gives a complex linear map $T$ on the tangent space at the identity element (the class of principal divisors) of Jac $R$. Let $g$ be the sum of the genera of the components of $Y$. The tangent space $T_{e}(\mathrm{Jac} R) \cong \mathbf{C}^{g}$ splits up as a direct sum of generalized eigenspaces of the linear map $T$. In general an eigenspace need not exponentiate to a compact subgroup of Jac $Y$. But in the case that $T$ satisfies a quadratic polynomial with two distinct integral roots, the eigenspaces are the tangent spaces at the identity of two sub-tori. For if the linear map $T$ has an integral root $r$, then $T-r I$ is the infinitesimal action of the endomorphism $C-r I$, where $r I$ is multiplication by $r$ in Jac $R$. Moreover $T-r I$ is a multiple of projection onto the other eigenspace. Call that eigenspace $E$. Since $T-r I$ must preserve the lattice $L$ of points which exponentiate to the identity in Jac $R$, the eigenspace $E$ is spanned by its intersection with $L$. So the eigenspace $E$ exponentiates to a torus. Such a torus is called a Prym-Tjurin variety. Prym varieties constitute a special case of Prym-Tjurin varieties in which the curve $R$ is a double cover of another curve and the correspondence is the graph of the deck transformation which exchanges the two sheets of the cover $R$. This notion of Prym is from Mumford. A more complete discussion of Prym varieties and integrable systems is in $[\mathrm{P}]$.

V. Kanev produces a set of correspondences on spectral curves. We generalize these and study them via group theory. Among our correspondences identified with the group algebra $\mathbf{Z}[W]$ are many which satisfy a quadratic polynomial.

Proposition 8. Let $\langle$,$\rangle be the inner product on \mathfrak{h}^{*}$ dual to the Killing form. Let $\lambda$ and $\alpha$ be any two weights. Then in $\mathbf{Z}[W]$ the element

$$
P_{\lambda, \alpha}=\sum_{w \in W}\left\langle w^{-1}(\lambda), \alpha\right\rangle w
$$

satisfies the quadratic polynomial $P_{\lambda, \alpha}^{2}=\left(\frac{|W|}{\operatorname{dim} \mathfrak{h}}\langle\lambda, \alpha\rangle\right) P_{\lambda, \alpha}$.

Proof. This follows from the orthogonality relation 4.5(ii) in [BD, p. 79] which says that for an irreducible representation $V$ of the group $W$ and $\lambda, \alpha, \beta, \gamma \in V$, one has

$$
\frac{1}{|W|} \sum_{w \in W}\left\langle w^{-1} \lambda, \alpha\right\rangle\langle w \beta, \gamma\rangle=\frac{1}{\operatorname{dim} V}\langle\beta, \alpha\rangle\langle\lambda, \gamma\rangle
$$


We use $V=\mathfrak{h}^{*}$, so $\operatorname{dim} V=\operatorname{dim} \mathfrak{h}$. Now compute.

$$
\left(P_{\lambda, \alpha}\right)^{2}=\sum_{w \in W} \sum_{z \in W}\left\langle w^{-1}(\lambda), \alpha\right\rangle w\left\langle z^{-1}(\lambda), \alpha\right\rangle z
$$

Replace $z$ by $u=w z$.

$$
\begin{aligned}
& =\sum_{w \in W} \sum_{u \in W}\left\langle w^{-1}(\lambda), \alpha\right\rangle\left\langle u^{-1} w(\lambda), \alpha\right\rangle u \\
& =\sum_{u \in W}\left(\sum_{w \in W}\left\langle w^{-1}(\lambda), \alpha\right\rangle\langle w(\lambda), u(\alpha)\rangle\right) u
\end{aligned}
$$

Apply the orthogonality relation.

$$
\begin{aligned}
& =\sum_{u \in W}\left(\frac{|W|}{\operatorname{dim} \mathfrak{h}}\langle\lambda, \alpha\rangle\langle\lambda, u(\alpha)\rangle\right) u \\
& =\frac{|W|}{\operatorname{dim} \mathfrak{h}}\langle\lambda, \alpha\rangle \sum_{u \in W}\left\langle u^{-1}(\lambda), \alpha\right\rangle u \\
& =\frac{|W|}{\operatorname{dim} \mathfrak{h}}\langle\lambda, \alpha\rangle P_{\lambda, \alpha} .
\end{aligned}
$$

Also since $W$ maps weights to weights and by definition $\langle\alpha, \beta\rangle \in \mathbf{Z}$ for any two weights, $P_{\lambda, \alpha}=\sum_{w \in W}\left\langle w^{-1}(\lambda), \alpha\right\rangle w$ is an element of $\mathbf{Z}[W]$.

The next lemma makes clear the action of $P_{\lambda, \alpha}$ on $\mathfrak{h}^{*}$.

Lemma 9. Let $\alpha, \beta, \gamma$, and $\lambda$ denote elements of $\mathfrak{h}^{*}$. Then

$$
P_{\lambda, \alpha}(\beta)=\frac{|W|}{\operatorname{dim} \mathfrak{h}}\|\alpha\|\left\|p r_{\alpha} \beta\right\| \lambda
$$

where $\mathrm{pr}_{\alpha} \beta$ means the orthogonal projection with respect to the Killing form onto the one-dimensional of span of $\alpha$ in $\mathfrak{h}^{*}$.

Proof.

$$
\begin{aligned}
\left\langle P_{\lambda, \alpha}(\beta), \gamma\right\rangle & =\left\langle\sum_{w \in W}\left\langle w^{-1}(\lambda), \alpha\right\rangle w(\beta), \gamma\right\rangle \\
& =\sum_{w \in W}\left\langle w^{-1}(\lambda), \alpha\right\rangle\langle w(\beta), \gamma\rangle \\
& =\frac{|W|}{\operatorname{dim} \mathfrak{h}}\langle\alpha, \beta\rangle\langle\lambda, \gamma\rangle
\end{aligned}
$$

where the last equality is the orthogonality relation.

Each $P_{\lambda, \alpha} \in \mathbf{Z}[W]$ gives a correspondence on $Y$ and hence an endomorphism of Jac $Y$ satisfying a quadratic polynomial of the form $P_{\lambda, \alpha}^{2}-K P_{\lambda, \alpha}=0$. The image of $P_{\lambda, \alpha}$ is therefore a Prym-Tjurin variety. However the previous lemma shows that the image of $P_{\lambda, \alpha}$ depends only on $\lambda$ and is independent of $\alpha$.

Notation. Let $\operatorname{Tur}_{\lambda} Y$ denote the image of any of the maps

$$
P_{\lambda, \alpha}=\sum_{w \in W}\left\langle w^{-1}(\lambda), \alpha\right\rangle w \in \mathbf{Z}[W]
$$

viewed as a map on the Jacobi variety of the master spectral curve, $P_{\lambda, \alpha}:$ Jac $Y \rightarrow$ $\operatorname{Jac} Y$. 
The notation "Tur" is used since later some actual Prym varieties will come up. For brevity we drop the letter $\mathrm{j}$ which indicates that the $\mathrm{T}$ in Tjurin's name is palatalized.

Proposition 10. The Prym-Tjurin varieties $\operatorname{Tur}_{\lambda} Y$ for the master curve, indexed by weights, are all isogenous.

Proof. The endomorphism $P_{\lambda, \alpha}$ of Jac $Y$ maps $\operatorname{Tur}_{\alpha} Y$ to $\operatorname{Tur}_{\lambda} Y$ and up to multiplication by an integer has an inverse $P_{\alpha, \lambda}$. In particular for $L \in \operatorname{Tur}_{\alpha} Y$ one has $P_{\alpha, \lambda} P_{\lambda, \alpha}(L)=\frac{|W|^{2}}{(\operatorname{dim} \mathfrak{h})^{2}}\|\alpha\|\|\lambda\| L$.

Now we examine elements in the Hecke rings that correspond to the $P_{\alpha, \lambda}$ 's. Recall that $S_{\lambda}$ denotes the stabilizer in $W$ of a weight $\lambda$ and $\pi_{\lambda}: Y \rightarrow Y / S_{\lambda}$ is the projection.

Definition. Suppose $S_{\gamma} \subset S_{\lambda}$. Let

$$
P_{\lambda}=P_{\lambda, \lambda}=\sum_{w \in W}\left\langle w^{-1} \lambda, \lambda\right\rangle w
$$

Define

$$
P_{\lambda}^{\gamma}=\left|S_{\gamma}\right| \sum_{w: S_{\gamma} \backslash W / S_{\gamma}}\left\langle w^{-1}(\lambda), \lambda\right\rangle S_{\gamma} w S_{\gamma} \in H\left(S_{\gamma}, W\right) .
$$

Note that $P_{\lambda}^{\gamma}=\left|S_{\gamma}\right| \sum_{w: S_{\gamma} \backslash W}\left\langle w^{-1}(\lambda), \lambda\right\rangle S_{\gamma} w \in \mathbf{Z}\left[S_{\gamma} \backslash W\right]^{S_{\gamma}}$. A representative of $P_{\lambda}^{\gamma}$ in $\mathbf{Z}[W]$ is $\overline{P_{\lambda}^{\gamma}}=P_{\lambda}$. Also note that the coefficients of $P_{\lambda}$ are constant on double cosets of $S_{\lambda}$ since $\left\langle s w s^{\prime} \lambda, \lambda\right\rangle=\left\langle w s^{\prime} \lambda, s^{-1} \lambda\right\rangle=\langle w \lambda, \lambda\rangle$. So $P_{\lambda} s=s P_{\lambda}=P_{\lambda}$ for $s \in S_{\gamma}$. By Proposition $7, P_{\lambda}^{\gamma}$ acts as a correspondence on $Y_{\gamma}$. This action is

$$
P_{\lambda}^{\gamma}(x)=\frac{1}{\left|S_{\gamma}\right|} \pi_{\gamma} P_{\lambda} \pi_{\gamma}^{-1}(x)
$$

Lemma 11. Suppose the stablizers of the weights $\beta, \gamma$, and $\lambda$ are nested: $S_{\beta} \subset$ $S_{\gamma} \subset S_{\lambda}$. Then the diagram of correspondences

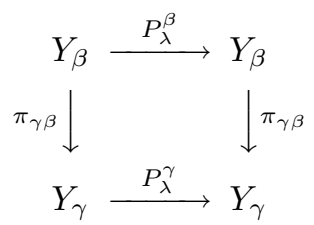

commutes.

Proof. Suppose that $S_{\theta} \subset S_{\lambda}$. If $\pi_{\theta}(x)=x_{\theta}$ then $\pi_{\theta}^{-1}\left(x_{\theta}\right)=\Sigma_{s \in S_{\theta}} s \cdot x$. So,

$$
\begin{aligned}
P_{\lambda}^{\theta}\left(x_{\theta}\right) & =\frac{1}{\left|S_{\theta}\right|} \pi_{\theta} P_{\lambda} \pi_{\theta}^{-1}\left(x_{\theta}\right) \\
& =\frac{1}{\left|S_{\theta}\right|} \pi_{\theta} P_{\lambda} \Sigma_{s \in S_{\theta}} s \cdot x \\
& =\pi_{\theta} P_{\lambda}(x) \text { since } P_{\lambda} \text { is } S_{\theta} \text { invariant }
\end{aligned}
$$

Let $\pi_{\beta}(x)=x_{\beta}$ and $\pi_{\gamma \beta}\left(x_{\beta}\right)=x_{\gamma}$. Then $\pi_{\gamma \beta} P_{\lambda}^{\beta}\left(x_{\beta}\right)=\pi_{\gamma \beta} \pi_{\beta} P_{\lambda}(x)=\pi_{\gamma} P_{\lambda}(x)$. Also, $P_{\lambda}^{\beta} \pi_{\gamma \beta}\left(x_{\beta}\right)=P_{\lambda}^{\beta}\left(x_{\gamma}\right)=\pi_{\gamma} P_{\lambda}(x)$. 
Theorem 12. $P_{\lambda}^{\gamma}$ defines a Prym-Tjurin variety in $\operatorname{Jac} Y_{\gamma}$ when $S_{\gamma} \subset S_{\lambda}$.

Proof. We check that $P_{\lambda}^{\gamma}$ satisfies the same quadratic polynomial that $P_{\lambda}$ does. $P_{\lambda}$ is constant on $S_{\gamma}$ orbits in $Y$. Also $\pi_{\gamma}^{-1} \pi_{\gamma}(x)=S_{\gamma} \cdot x$, the $S_{\gamma}$ orbit of $x \in Y$. Hence $P_{\lambda} \pi_{\gamma}^{-1} \pi_{\gamma}(x)=\left|S_{\gamma}\right| P_{\lambda}(x)$. Therefore $\left(P_{\lambda}^{\gamma}\right)^{2}(x)=\frac{1}{\left|S_{\gamma}\right|} \pi_{\gamma} P_{\lambda}^{2} \pi_{\gamma}^{-1}(x)$. This shows that $P_{\lambda}^{\gamma}$ satisfies the same quadratic polynomial as $P_{\lambda}$, namely $P^{2}+\frac{|W|}{\operatorname{dim} \mathfrak{h}}\|\lambda\|^{2} P=0$, and so defines a Prym-Tjurin variety.

Denote the Prym-Tjurin variety defined as the image of $P_{\lambda}^{\gamma}$ in $\operatorname{Jac}_{\gamma}$ as $\operatorname{Tur}_{\lambda}\left(Y_{\gamma}\right)$.

Theorem 13. The Prym-Tjurin varieties $\operatorname{Tur}_{\lambda}\left(Y_{\gamma}\right)$ for the various $\lambda$ and $\gamma$ with $S_{\gamma} \subset S_{\lambda}$ are all isogenous. In fact if $S_{\beta} \subset S_{\gamma} \subset S_{\lambda}$ then $\pi_{\gamma \beta}^{*}: \operatorname{Jac} X_{\gamma} \rightarrow X_{\beta}$ restricts to an isogeny $\operatorname{Tur}_{\lambda}\left(X_{\gamma}\right) \rightarrow \operatorname{Tur}_{\lambda}\left(X_{\beta}\right)$.

Proof. Since it has already been shown that the Prym-Tjurin varieties $\operatorname{Tur}_{\lambda} Y$ for the master curve are all isogenous, it is only necessary to show that the varieties $\operatorname{Tur}_{\lambda}\left(Y_{\gamma}\right)$ are all isogenous for a fixed weight $\lambda$.

Since $\operatorname{Tur}_{\lambda}\left(Y_{\gamma}\right)$ is the image in $\operatorname{Jac} Y_{\gamma}$ of $P_{\lambda}^{\gamma}$, any element of $\operatorname{Tur}_{\lambda}\left(Y_{\gamma}\right)$ is the equivalence class of a divisor of the form $P_{\lambda}^{\gamma} \mathcal{D}$ for $\mathcal{D}$ a divisor on $Y_{\gamma}$. For any divisor $\mathcal{D}$ on $Y_{\gamma}$ we have $\pi^{*}\left[P_{\lambda}^{\gamma} \mathcal{D}\right] \in \operatorname{Tur}_{\lambda}\left(Y_{\beta}\right)$ since by Lemma $11, \pi_{\gamma \beta}^{-1} P_{\lambda}^{\gamma} \mathcal{D}=P_{\lambda}^{\beta} \pi_{\gamma \beta}^{-1} \mathcal{D}$.

This shows that $\pi_{\gamma \beta}^{*}$ maps $\operatorname{Tur}_{\lambda} Y_{\gamma}$ into $\operatorname{Tur}_{\lambda} Y_{\beta}$. Recall that by Proposition 3, $\pi_{\gamma \beta}^{*}$ has a finite kernel as a map from $\operatorname{Jac} Y_{\gamma}$ to $\operatorname{Jac} Y_{\beta}$, so to show $\pi_{\gamma \beta}^{*}$ is an isogeny of $\operatorname{Tur}_{\lambda}\left(Y_{\gamma}\right)$ with $\operatorname{Tur}_{\lambda} Y_{\beta}$, it remains to show $\pi_{\gamma \beta}^{*}$ maps onto $\operatorname{Tur}_{\lambda} Y_{\beta}$. It is enough to show for any $x_{\beta} \in Y_{\beta}$ that $P_{\lambda}^{\beta}\left(x_{\beta}\right) \in \operatorname{Im} \pi_{\gamma \beta}^{-1} P_{\lambda}^{\gamma}$. Let $x \in Y$ satisfy $\pi_{\beta}(x)=x_{\beta}$. Then by Lemma 11, $P_{\lambda}^{\beta}\left(x_{\beta}\right)=\pi_{\beta} P_{\lambda}(x)$. Now $\pi_{\gamma \beta}^{-1} \pi_{\gamma}(x)=\frac{1}{\left|S_{\beta}\right|} \pi_{\beta} \sum_{s \in S_{\gamma}} s(x)$ and $\pi_{\gamma}^{-1} \pi_{\gamma \beta}\left(x_{\beta}\right)=\sum_{s \in S_{\gamma}} s(x)$. So,

$$
\begin{aligned}
\pi_{\gamma \beta}^{-1} P_{\lambda}^{\gamma}\left(\pi_{\gamma \beta}\left(x_{\beta}\right)\right) & =\frac{1}{\left|S_{\gamma}\right|}\left(\pi_{\gamma \beta}^{-1} \pi_{\gamma}\right) P_{\lambda}\left(\pi_{\gamma}^{-1} \pi_{\gamma \beta}\right)\left(x_{\beta}\right) \\
& =\frac{1}{\left|S_{\gamma}\right|\left|S_{\beta}\right|} \pi_{\beta}\left(\sum_{s \in S_{\gamma}} s\right) P_{\lambda}\left(\sum_{s \in S_{\gamma}} s\right)(x) \\
& =\frac{\left|S_{\gamma}\right|}{\left|S_{\beta}\right|} \pi_{\beta} P_{\lambda}(x)=\frac{\left|S_{\gamma}\right|}{\left|S_{\beta}\right|} P_{\lambda}^{\beta}\left(x_{\beta}\right) .
\end{aligned}
$$

Therefore $\operatorname{im} P_{\lambda}^{\beta} \subset \operatorname{im} \pi_{\gamma \beta}^{*} P_{\lambda}^{\gamma}$ since $\operatorname{im} \pi_{\gamma \beta}^{*} P_{\lambda}^{\gamma}$ is a torus and so a divisible group. Thus $\pi_{\gamma \beta}^{*} \operatorname{maps} \operatorname{Tur}_{\lambda}\left(Y_{\gamma}\right)$ onto $\operatorname{Tur}_{\lambda}\left(Y_{\beta}\right)$.

Note that if $\mu$ is onto $W$ then Proposition 4 implies that the kernel of $\pi^{*}: \operatorname{Tur}_{\lambda}\left(Y_{\gamma}\right)$ $\rightarrow \operatorname{Tur}_{\lambda}\left(Y_{\beta}\right)$ is a sum of $\mathbf{Z}_{2}^{\prime} s$.

\section{Dimension of the Prym-TuUrin VARIEty}

This section finds a Lefschetz type formula for the dimension of $\operatorname{Tur}_{\lambda}\left(Y_{\gamma}\right)$.

Since $\mathfrak{h}$ is the complexification of a real representation, $\mathfrak{h} \cong \mathfrak{h}^{*}$ as $\mathbf{C}[W]$ modules. In the following the inner product notation $\langle$,$\rangle will be used on the space of$ representations of $W$ with the irreducible representations forming an orthonormal basis.

Lemma 14. Suppose $\alpha \in \mathfrak{h}^{*}$. Then $\left\{P_{\lambda, \alpha} \mid \lambda \in \mathfrak{h}^{*}\right\} \cong \mathfrak{h}^{*}$ as $\mathbf{C}[W]$ modules. 
Proof. One can check that $a P_{\lambda, \alpha}+b P_{\delta, \alpha}=P_{a \lambda+b \delta, \alpha}$ and that $w \cdot P_{\lambda, \alpha}=P_{w \cdot \lambda, \alpha}$. The isomorphism is $\lambda \rightarrow P_{\lambda, \alpha}$.

A $\mathbf{C}[W]$ module $M$ decomposes as a sum $M=\underset{\chi \text { irr. }}{\bigoplus} k_{\chi} V_{\chi}$ of irreducible modules, write $M_{\chi}$ for the $\chi$-type $k_{\chi} V_{\chi} \subset M$.

Proposition 15. Let $M$ be a $\mathbf{C}[W]$ module.

(1) If $M=\bigoplus_{\chi \text { irr. }} M_{\chi}$ is its decomposition into $\chi$-types according to the irreducible representations of $W$, then $P_{\lambda, \alpha} M \subset M_{\mathfrak{h}}$. In particular, $T_{e} \operatorname{Tur}_{\lambda} Y \subset$ $\left(T_{e} \mathrm{Jac} Y\right)_{\mathfrak{h}}$, where $T_{e}$ means the tangent space at the identity.

(2) The dimension of $P_{\lambda, \alpha} M$ is $\langle\mathfrak{h}, M\rangle$.

Proof. Suppose $V$ is an irreducible summand of $M$. For any $v \in V$, the submodule $\mathbf{C}[W] P_{\lambda, \alpha} v$ is either $V$ or 0 . But $\mathbf{C}[W] P_{\lambda, \alpha}$ is isomorphic to $\mathfrak{h}^{*} \cong \mathfrak{h}$. Therefore if $V \nsubseteq \mathfrak{h}$ then $P_{\lambda, \alpha} V=0$. If $V \cong \mathfrak{h}$ then by Lemma $9, P_{\lambda, \alpha}$ is projection onto a one-dimensional subspace. Therefore, $P_{\lambda, \alpha} M=\langle\mathfrak{h}, M\rangle$.

Lemma 16. The dimension of the Prym-Tjurin variety is

$$
\operatorname{dim} \operatorname{Tur}_{\lambda}(Y)=\frac{1}{2}\left\langle\mathfrak{h}, H^{1}(Y ; \mathbf{C})\right\rangle .
$$

Proof. The action of $W$ as endomorphisms of the Jacobi variety Jac $Y$ gives a representation of $W$ on the cohomology group $H^{1}(Y, \mathbf{C})=H^{1,0}(Y) \oplus H^{0,1}(Y)$. Since $W$ acts on $Y$, the representations on the two summands are conjugate. Furthermore the tangent space of the Jacobi variety is $T_{e} \mathrm{Jac} Y \cong H^{0,1}(Y)$. Since $\mathfrak{h}$ arises from a real representation, $\left\langle H^{0,1}(Y), \mathfrak{h}\right\rangle=\left\langle H^{1,0}(Y), \mathfrak{h}\right\rangle=\frac{1}{2}\left\langle H^{1}(Y, \mathbf{C}), \mathfrak{h}\right\rangle$. The lemma then follows from the preceding proposition.

The next result computes the dimension of $\operatorname{Tur}_{\lambda}$ more explicitly.

Theorem 17. Suppose $\mu: \pi_{1}\left(P_{*}\right) \rightarrow W$ has image $M$. For $x \in Y$, let $I_{x} \subset W$ be the subgroup of deck transformations that fix $x$, and let $\mathfrak{h}^{I_{x}}$ be the fixed point set in $\mathfrak{h}$ of the action of $I_{x}$. Then

$$
\begin{aligned}
\left\langle\mathfrak{h}, H^{1}(Y ; \mathbf{C})\right\rangle= & \sum\left(\operatorname{dim} \mathfrak{h}-\operatorname{dim} \mathfrak{h}^{I_{x}}\right) \\
& -(\operatorname{dim} \mathfrak{h})(\text { Euler char of } P)+2\langle\mathfrak{h}, \mathbf{C}[W / M]\rangle
\end{aligned}
$$

where the sum is taken over the ramification points of $\pi: Y \rightarrow P$ and for each ramification point $p \in P$, a choice $x \in \pi^{-1}(p)$ has been made.

Note that $\langle\mathfrak{h}, \mathbf{C}[W / M]\rangle=\left\langle\operatorname{res}_{M}^{W} \mathfrak{h}, 1_{M}\right\rangle$. If $\mu$ is an onto map, i.e., $W=M$, then $\langle\mathfrak{h}, \mathbf{C}[W / M]\rangle=0$. The proof below shows the validity of the formula for any irreducible representation $\chi$, not just $\chi=\mathfrak{h}$.

Proof. The master curve $Y$ is a branched $W$ bundle over the parameter space $P$. Let $\pi: Y \rightarrow P$ be the covering projection. Express $P$ as a simplicial complex such that the set of 0 -cells $P^{0}$ contains all the branch points of $\pi$. Let $P^{1}$ be the 1 -cells and $P^{2}$ the 2 -cells. Similarly express $Y$ as a simplicial complex with $Y^{i}=\pi^{-1}\left(P^{i}\right), \quad i=0,1,2$. With these cells form the following singular complex $\Delta(Y)$ with coefficients in $\mathbf{C}$.

$$
0 \stackrel{\partial_{3}}{\longrightarrow} \Delta_{2}(Y) \stackrel{\partial_{2}}{\longrightarrow} \Delta_{1}(Y) \stackrel{\partial_{1}}{\longrightarrow} \Delta_{0}(Y) \stackrel{\partial_{0}}{\longrightarrow} 0 .
$$

This chain complex is equivariant under the $W$ action on $Y$. 
If $V_{\chi}$ is irreducible and $\psi: V_{\chi} \rightarrow N$ is a map of $W$-modules, then $\psi$ is either zero or one-to-one, so the complex $\Delta(Y)$ can be written as a sum of complexes $\Delta(Y)_{\chi}$ of $\chi$-types:

$$
0 \stackrel{\left(\partial_{3}\right)_{\chi}}{\longrightarrow}\left(\Delta_{2}(Y)\right)_{\chi} \stackrel{\left(\partial_{2}\right)_{\chi}}{\longrightarrow}\left(\Delta_{1}(Y)\right)_{\chi} \stackrel{\left(\partial_{1}\right)_{\chi}}{\longrightarrow}\left(\Delta_{0}(Y)\right)_{\chi} \stackrel{\left(\partial_{0}\right)_{\chi}}{\longrightarrow} 0
$$

and moreover

$$
\frac{\operatorname{ker}\left(\partial_{i}\right)_{\chi}}{\operatorname{im}\left(\partial_{i}\right)_{\chi}}=H_{i}(Y)_{\chi}
$$

From the Euler characteristic of $(\Delta(Y))_{\chi}$ we get

$$
\begin{aligned}
& \left\langle\Delta_{0}(Y), \chi\right\rangle-\left\langle\Delta_{1}(Y), \chi\right\rangle+\left\langle\Delta_{2}(Y), \chi\right\rangle \\
& \quad=\left\langle H_{0}(Y ; \mathbf{C}), \chi\right\rangle-\left\langle H_{1}(Y ; \mathbf{C}), \chi\right\rangle+\left\langle H_{2}(Y ; \mathbf{C}), \chi\right\rangle .
\end{aligned}
$$

But if $X$ is an irreducible component of $Y=\cup_{w: W / M} w \cdot X$ then

$$
\begin{aligned}
H_{0}(Y ; \mathbf{C}) & =\mathbf{C}[W] \otimes_{M} H_{0}(X ; \mathbf{C}) \\
& =\operatorname{ind}_{M}^{W} 1_{M} \\
& =\mathbf{C}[W / M] .
\end{aligned}
$$

Here 1 denotes the trivial representation and $\operatorname{ind}_{M}^{W} \chi$ is the induced representation from the group $M$ to $W$. Similarly, $H_{2}(Y)=\operatorname{ind}_{M}^{W} 1_{M}$. Therefore

$$
\left\langle\Delta_{0}(Y), \chi\right\rangle-\left\langle\Delta_{1}(Y), \chi\right\rangle+\left\langle\Delta_{2}(Y), \chi\right\rangle=2\left\langle\operatorname{ind}_{M}^{W} 1_{M}, \chi\right\rangle-\left\langle H_{1}(Y ; \mathbf{C}), \chi\right\rangle .
$$

Now each 1-cell or 2-cell in $P$ lifts to $|W|$ cells in $Y$, so $\Delta_{2}(Y)=\mathbf{C}[W] \otimes_{\mathbf{C}} \Delta_{2}(P)$ and $\Delta_{1}(Y)=\mathbf{C}[W] \otimes_{\mathbf{C}} \Delta_{1}(P)$ while $\Delta_{0}(Y)=\oplus_{p_{i} \in P^{0}} R_{p_{i}}$ where $R_{p_{i}}=\mathbf{C}\left[W / I_{x_{i}}\right]=$ $\operatorname{ind}_{I_{x_{i}}}^{W} 1$ for $I_{x_{i}}$ the isotropy group of $x_{i} \in \pi^{-1}\left(p_{i}\right)$. In particular, if $p_{i}$ is not a branch point then $R_{p_{i}}=\mathbf{C}[W]$. Consequently we have the formulas

$$
\begin{aligned}
& \left\langle\Delta_{2}(Y), \chi\right\rangle=\left|P^{2}\right| \cdot\langle\mathbf{C}[W], \chi\rangle=\left|P^{2}\right| \operatorname{dim} \chi, \\
& \left\langle\Delta_{1}(Y), \chi\right\rangle=\left|P^{1}\right| \operatorname{dim} \chi, \\
& \left\langle\Delta_{0}(Y), \chi\right\rangle=\sum_{p \in P^{0}}\left\langle R_{p}, \chi\right\rangle .
\end{aligned}
$$

The first two of these formulas give

$($ Euler char of $P)(\operatorname{dim} \chi)=\left\langle\Delta_{2}(Y), \chi\right\rangle-\left\langle\Delta_{1}(Y), \chi\right\rangle+(\operatorname{dim} \chi)\left|P^{0}\right|$.

So,

$2\left\langle\operatorname{ind}_{M}^{W} 1, \chi\right\rangle-\left\langle H_{1}(Y ; \mathbf{C}), \chi\right\rangle=(\operatorname{dim} \chi)($ Euler char of $P)+\sum_{p \in P^{0}}\left(\left\langle R_{p}, \chi\right\rangle-\operatorname{dim} \chi\right)$.

But if $p$ is not a branch point then $\left\langle R_{p}, \chi\right\rangle=\operatorname{dim} \chi$, so the last sum need only be over the branch points of $\pi: Y \rightarrow P$.

Now $\left\langle\chi, R_{p}\right\rangle=\left\langle\chi, \operatorname{ind}_{I_{x}}^{W} 1\right\rangle=\left\langle\operatorname{res}_{I_{x}}^{W} \chi, 1\right\rangle$ by Frobenius reciprocity. Here $\operatorname{res}_{I_{x}}^{W} \chi$ is the restriction of $W$ acting by $\chi$ to a representation of the subgroup $I_{x}$. Write $V_{\chi}$ for the space the representation $\chi$ acts on. Then $\left\langle\operatorname{res}_{I_{x}}^{W} \chi, 1\right\rangle=\operatorname{dim}\left(V_{\chi}\right)^{I_{x}}$, the dimension of the fixed point set of $I_{x}$.

Therefore,

$2\left\langle\operatorname{ind}_{M}^{W} 1, \chi\right\rangle-\left\langle H_{1}(Y ; \mathbf{C}), \chi\right\rangle=(\operatorname{dim} \chi)($ Euler char of $P)+\sum\left(\operatorname{dim}\left(V_{\chi}\right)^{I_{x}}-\operatorname{dim} \chi\right)$ 
where the sum is over the ramification points of $\pi$ and for each ramification point $p$ a choice of $x \in \pi^{-1}(p)$ is made. Specialization to the representation of $W$ on $\mathfrak{h}$ gives the proposition.

Recall that the principal $W$ bundle $Y_{*} \rightarrow P_{*}$ is determined by the map $\mu$ : $\pi_{1}\left(P_{*}\right) \rightarrow W$. Let $p \in P$ be a ramification point of $\pi: Y \rightarrow P$ and let $x \in \pi^{-1}(P)$. Let $D \subset P$ be a disk containing $p$ but no other ramification points and whose boundary $\partial D$ is a based loop in $P_{*}$. Then $\pi^{-1}(D)$ consists of $\left|W / I_{x}\right|$ disjoint copies of $D$, i.e. $D_{1} \cup D_{2} \cup \cdots \cup D_{\left|W / I_{x}\right|}$. Suppose $x \in D_{k}$. Then $\pi: D_{k} \backslash\{x\} \rightarrow D \backslash\{p\}$ is a regular covering space with group $I_{x}$. Since $D \backslash\{p\}$ is homotopic to a circle, the only covers are cyclic. Since the isotropy group is cyclic the fixed point set $V_{\chi}^{I_{x}}$ is the same as the fixed point set of a generator of $I_{x}$.

Suppose $D_{1}$ is the component that contains the basepoint of $Y$ and $z \cdot D_{k}=D_{1}$ where $z \in W$. Let $l$ be the loop of degree 1 about $\partial D$. Then $\mu(l)=w \in W$ is the generator for the deck transformations of $\pi: D_{1} \backslash\{z \cdot x\} \rightarrow D \backslash\{p\}$. So $I_{x}$ is the cyclic group generated by $z^{-1} w z$. The determination of $z$ varies with the choice of basepoints. We have shown the following.

Lemma 18. Let $p \in P$ be a ramification point of $\pi: Y \rightarrow P$ and $x \in \pi^{-1}(p)$ a point in the master curve $Y$. Let $I_{x} \subset W$ be the isotropy group of $x$. Then $I_{x}$ is cyclic. Furthermore, $I_{x}$ is conjugate to the subgroup $\langle w\rangle$ where $w$ is the image in $W$ of a loop about the point $p$.

Suppose that $\mu$ maps onto $W$ so that $Y_{*} \rightarrow P_{*}$ is a connected covering space. We want to determine $I_{x}$ up to conjugacy and do so using the monodromy representation of $W$. Let $F$ be the fiber over the basepoint in $P_{*}$. The group $W$ acts on $F$ on the left as deck transformations and $\pi_{1}\left(P_{*}\right)$ acts on the right via the monodromy representation $[\mathrm{M}]$. The monodromy representation factors through $W$ and so defines a right action of $W$ on $F$ (see [MS, p. 140]). It can be used to determine $\mu$. This factorization depends on the basepoint in $F$. A change of this basepoint alters the vertical map below by an inner automorphism of $W$.

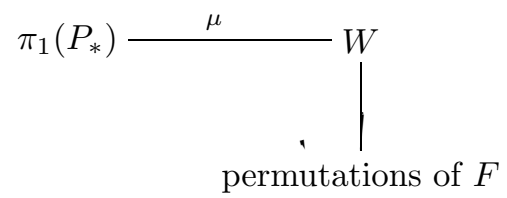

While deck transformations act only on $Y_{*}$ there is a monodromy representation from any of the covering spaces $Y_{\lambda *}$. Use the basepoint in $Y_{*}$ to identify the fiber $F$ of the master curve $Y_{*}$ with the group $W$. Then the fiber $\pi_{\lambda}(F)$ of the curve $Y_{*} / S_{\lambda}$ may be identified with the inverse images of its points, which are the right cosets $\left\{S_{\lambda} w\right\}$ since the group of deck transformations acts on the left. The action of $\pi_{1}\left(P_{*}\right)$ via the monodromy representation on the fiber $\pi_{\lambda}(F)$ factors through the right action of $W$ on the right cosets $\left\{S_{\lambda} w\right\}$. The image of $\pi_{1}\left(P_{*}\right)$ in the permutation group of a fiber of $Y_{\lambda *}$ is called the monodromy group of the cover.

Proposition 19. If $\mu$ is onto $W$ then the monodromy group of the cover $Y_{\lambda *} \rightarrow P_{*}$ is $W$ for any nonzero weight $\lambda$.

We identify the monodromy group with $W$.

Proof. Proposition 19 of Paper I says in this setting that the monodromy group of $Y_{*} / S_{\lambda}$ is $W / N$ where $N$ is the largest normal subgroup of $W$ contained in $S_{\lambda}$. 
We just need to show that $N=\{e\}$. Suppose $w \in N$. Then $w \cdot \lambda=\lambda$ since $N \subset S_{\lambda}$, the stabilizer of the weight $\lambda$. Since $N$ is normal, for any $w_{1} \in W$ one has $w_{1} w w_{1}^{-1} \in N \subset S_{\lambda}$ and so $w_{1} w w_{1}^{-1} \cdot \lambda=\lambda$ or $w \cdot\left(w_{1}^{-1} \lambda\right)=w_{1}^{-1} \lambda$. So $w$ stabilizes the entire $W$ orbit of $\lambda$ which spans $\mathfrak{h}^{*}$. Therefore $w$ fixes all of $\mathfrak{h}^{*}$ and must be the identity element $e \in W$.

This proposition says we can calculate the conjugacy class of $I_{x}$ using the monodromy representation of any spectral curve whenever $\mu$ maps onto $W$. For the infinite families of Lie algebras the right $W$ action on a fiber of the spectral curve obtained from the classical representation of the Lie algebra is itself a standard action of $W$ on a set.

In order to do calculations involving Weyl groups we establish our conventions for permutation groups and signed permutation groups. In accordance with viewing permutations as functions we multiply right to left so that $(1,2)(2,3)=(1,2,3)$. Then $\Sigma_{n}$ acts on the left as permutations of $\{1,2, \ldots, n\}$. Thus we write the image of 1 under $\sigma=(1,2,3)$ as $\sigma(1)=2$. Write $t_{i}$ for the element that interchanges $i$ and $-i$ in the action of the signed permutation group $\mathbf{Z}_{2}^{n} \rtimes \Sigma_{n}$ on the set $\{1,2, \ldots, n,-1,-2, \ldots,-n\}$. For $\sigma \in \Sigma_{n}$ we have $\sigma t_{i} \sigma^{-1}=t_{\sigma(i)}$. The actions of these groups on the sets $\{i \mid 1 \leq i \leq n\}$ or $\{ \pm i \mid 1 \leq i \leq n\}$ now agree with the action of $W$ on the subsets $\left\{e_{i} \mid 1 \leq i \leq n\right\}$ or $\left\{ \pm e_{i} \mid 1 \leq i \leq n\right\}$ of $\mathfrak{h}^{*}$ or a convenient ambient space for $\mathfrak{h}^{*}$ for the respective Lie algebra [H2, p. 64]. Now we examine cases.

type $A_{n} \cdot W=\Sigma_{n+1}$, the symmetric group on $n+1$ symbols. For the classical representation $S_{\lambda}=\Sigma_{n}$, the permutations of the last $n$ symbols.

The fiber of the spectral curve from the classical representation of $\operatorname{sl}(n+1, \mathbf{C})$ is $S_{\lambda} \backslash W \cong \Sigma_{n} \backslash \Sigma_{n+1}$ which consists of $n+1$ cosets. The cosets are $R_{k}=\Sigma_{n}(1, k)=$ $\{\sigma \mid \sigma(k)=1\}$. The right $W$ action is then $R_{k} \sigma=R_{\sigma^{-1}(k)}$. In this manner $W$ acts as the full permutation group on right cosets.

type $B_{n}, C_{n} . W=\mathbf{Z}_{2}^{n} \rtimes \Sigma_{n}$, the full signed permutation group on $\{ \pm 1, \pm 2, \ldots, \pm n\}$. For the classical representation $S_{\lambda}=\mathbf{Z}_{2}^{n-1} \rtimes \Sigma_{n-1}$, the signed permutation group on $\{ \pm 2, \ldots, \pm n\}$. The cosets in $S_{\lambda} \backslash W=\mathbf{Z}_{2}^{n-1} \rtimes \Sigma_{n-1} \backslash \mathbf{Z}_{2}^{n} \rtimes \Sigma_{n}$ are $\left\{R_{\epsilon k} \mid \epsilon=\right.$ $\pm 1,1 \leq k \leq n\}$ where $R_{\epsilon k}=S_{\lambda} t_{1}^{\epsilon}(1, k)=\{\sigma \mid \sigma(\epsilon k)=1\}$. The Weyl group $W$ acts on the right by $R_{\epsilon k} \cdot \sigma=R_{\sigma^{-1}(\epsilon k)}$.

type $D_{n} \cdot W=\mathbf{Z}_{2}^{n-1} \rtimes \Sigma_{n}$, the group of signed permutations on $\{ \pm 1, \pm 2, \ldots, \pm n\}$ with only even numbers of sign changes. For the classical representation $S_{\lambda}=$ $\mathbf{Z}_{2}^{n-2} \rtimes \Sigma_{n-1}$, the group of signed permutations on $\{ \pm 2, \ldots, \pm n\}$ with even numbers of sign changes. The set of right cosets $S_{\lambda} \backslash W$ is $\left\{R_{\epsilon k} \mid \epsilon= \pm 1,1 \leq k \leq n\right\}$ where $R_{\epsilon k}=S_{\lambda}\left(t_{1} t_{2}\right)^{\epsilon}(1, k)=\{\sigma \mid \sigma(\epsilon k)=1\}$. Again $R_{\epsilon k} \cdot \sigma=R_{\sigma^{-1}(\epsilon k)}$.

type $G_{2} \cdot W=\mathbf{Z}_{2} \times \Sigma_{3}$. Write $t$ for the generator of the $\mathbf{Z}_{2}$ summand. There are two fundamental weights which, following the notation of [H2], are designated $\lambda_{1}=(0,-1,1)$ and $\lambda_{2}=(-1,-1,2)$ in the $e_{i}$ basis. In some other sources such as [BMP] the subscripts on the names for the weights are reversed. The two conjugacy classes of proper parabolic subgroups are represented by the two subgroups $S_{\lambda_{1}}=$ $\langle t(2,3)\rangle$ and $S_{\lambda_{2}}=\langle(1,2)\rangle$, both cyclic of order 2. Paper I has a misstatement, and should have said that the conjugacy class of $\langle(1,2)\rangle$ is associated with the longer, rather than shorter, root. 
Each right coset in $S_{\lambda_{1}} \backslash W$ can be identified by the unique element of $\Sigma_{3}$ it contains. Write $R_{e}=\{e, t(2,3)\}, R_{12}=\{(1,2), t(1,3,2)\}, R_{13}=\{(1,3), t(1,2,3)\}, R_{23}$ $=\{(2,3), t\}, R_{123}=\{(1,2,3), t(1,3)\}$, and $R_{132}=\{(1,3,2), t(1,2)\}$. The following table gives the representation of the right action of $W$ on the cosets as permutations of the cosets.

$\begin{array}{cc}e & \text { identity } \\ (1,2) & \left(R_{e}, R_{12}\right)\left(R_{13}, R_{123}\right)\left(R_{23}, R_{132}\right) \\ (1,3) & \left(R_{e}, R_{13}\right)\left(R_{12}, R_{132}\right)\left(R_{23}, R_{123}\right) \\ (2,3) & \left(R_{e}, R_{23}\right)\left(R_{12}, R_{123}\right)\left(R_{13}, R_{132}\right) \\ (1,2,3) & \left(R_{e}, R_{123}, R_{132}\right)\left(R_{12}, R_{13}, R_{23}\right) \\ (1,3,2) & \left(R_{e}, R_{132}, R_{123}\right)\left(R_{12}, R_{23}, R_{13}\right) \\ t & \left(R_{e}, R_{23}\right)\left(R_{12}, R_{132}\right)\left(R_{13}, R_{123}\right) \\ t(1,2) & \left(R_{e}, R_{132}\right)\left(R_{12}, R_{23}\right) \\ t(1,3) & \left(R_{e}, R_{123}\right)\left(R_{13}, R_{23}\right) \\ t(2,3) & \left(R_{12}, R_{13}\right)\left(R_{123}, R_{132}\right) \\ t(1,2,3) & \left(R_{e}, R_{13}, R_{132}, R_{23}, R_{123}, R_{12}\right) \\ t(1,3,2) & \left(R_{e}, R_{12}, R_{123}, R_{23}, R_{132}, R_{13}\right)\end{array}$

Note that the cycle structure of the action of $w \in W$ on the cosets picks out the conjugacy class of $w$ except for distinguishing between $t$ and the class of 2-cycles $\{(1,2),(1,3),(2,3)\}$. However the action of $t$ on a fiber in the spectral curve $Y_{\lambda_{1}}$ is easy to recognize since it flips the sign on the $z$ coordinate that gives the eigenvalue.

For the other parabolic subgroup the situation is like that of types $B_{n}, C_{n}$, and $D_{n}$ above. $W=\mathbf{Z}_{2} \times \Sigma_{3} . S_{\lambda_{2}}=\Sigma_{2}$ is contained in the $\Sigma_{3}$ summand of $W$ and is generated by $(1,2)$. Treat $W$ as a subgroup of the signed permutation group on $\{ \pm 1, \pm 2, \pm 3\}$. The cosets in $S_{\lambda} \backslash W=\Sigma_{2} \backslash \mathbf{Z}_{2} \times \Sigma_{3}$ are $\left\{R_{\epsilon k} \mid \epsilon= \pm 1,1 \leq k \leq 3\right\}$ where $R_{\epsilon k}=S_{\lambda} t^{\epsilon}(3, k)=\{\sigma \mid \sigma(\epsilon k)=3\}$. Once again the Weyl group $W$ acts on the right by $R_{\epsilon k} \cdot \sigma=R_{\sigma^{-1}(\epsilon k)}$.

Specific computations use realizations of the Weyl groups for the algebras of types $B_{n}, C_{n}, D_{n}$, and $G_{2}$ as signed permutations acting on a fiber of the classical curve or, in the case of $G_{2}$, a fiber of $Y_{\lambda_{2}}$. Here we are considering $W\left(D_{n}\right) \subset \mathbf{Z}_{2}^{n} \rtimes \Sigma_{n}$, the signed permutations on $n$ pairs and $W\left(G_{2}\right) \subset \mathbf{Z}_{2}^{3} \times \Sigma_{3}$. The points in the fiber over $s_{0} \in P$ have coordinates $\left(s_{0}, z_{i}\right)$ for $i=1, \ldots, 2 n$. The set $\mathcal{Z}=\left\{z_{i} \mid i=\right.$ $1, \ldots, 2 n\}$ can be split into pairs of points since if $z \in \mathcal{Z}$ then $-z \in \mathcal{Z}$ and each $z$ occurs uniquely for $s_{0}$ not a ramification point. The sign changes in the signed permutation groups do act as sign changes on the $z$-coordinate as we now explain. The map $A: Y_{\lambda_{1} *} \rightarrow\left(G / H \times \mathfrak{h}_{*}\right) / S_{\lambda_{1}}$ given by $A(s, z)=[(g H, h)]$ is partially characterized by $\lambda(h)=z$ (see [MS]). The action of $\tau=\prod_{i=1}^{n} t_{i} \in W\left(B_{n}\right)$, or $W\left(C_{n}\right)$, is demonstrated in the diagram below, in which $n$ represents $\tau$ as a coset in $W=N_{H} / C_{H}$.

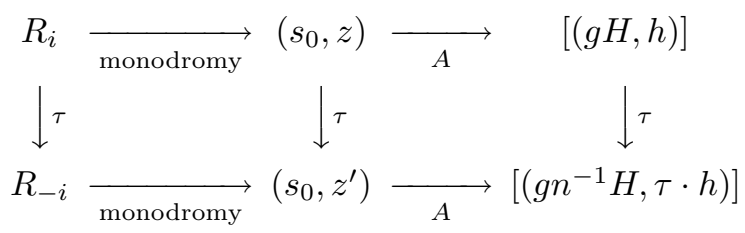

We have $z=\lambda(h)$ while $z^{\prime}=\lambda(\tau \cdot h)=-\lambda(h)=-z$.

For the algebras $B_{n}$ and $C_{n}$ the \pm labelling of the pairs and the assignment of a number from $1, \ldots, n$ to each pair can be chosen in any manner. A change 
in labelling can be accomplished through an inner automorphism of $W$. For the algebras $D_{n}$ and $G_{2}$ this is not the case, but changes can still be made through the full signed permutation group. Conveniently the representations we consider for $D_{n}$ and the $\lambda_{2}$ case for $G_{2}$ extend to representations of the full signed permutation group on $\mathfrak{h}_{D_{n}}^{*} \cong \mathbf{C}^{n}$ or $\mathfrak{h}_{G_{2}}^{*} \oplus \mathbf{C} \cong \mathbf{C}^{3}$. Consequently any labelling will allow computation of the dimensions of the fixed point sets $\mathfrak{h}^{I_{x}}$, as different labellings still differ by an automorphism of $W$. Inspection shows that this is also valid for the $\lambda_{1}$ case of $G_{2}$.

\section{When Tur $Y$ IS A JACOBI OR PRYM VARIETY}

This section pins down the Prym-Tjurin varietes for the case of the classical Lie algebras. Theorem 13 showed that for a given initial condition $A: P \rightarrow \mathfrak{g}$ all the Prym-Tjurin varieties $\operatorname{Tur}_{\lambda}\left(Y_{\gamma}\right)$ are isogenous for the various choices of a spectral curve $Y_{\gamma}$ and a weight $\lambda$ with $S_{\gamma} \subset S_{\lambda}$. For Lie algebas of type $B, C$, or $D$ the Prym-Tjurin variety is, up to isogeny, a Prym variety. The classical spectral curve is a double cover of a quotient curve of $Y$. Although the quotient curve is a quotient of the master curve $Y$ by a subgroup of the Weyl group, it is not a spectral curve. The Prym-Tjurin is the Prym variety of this double cover. For type $A$, we have in the special, but common, case where the parameter space is the Riemann sphere that the Prym-Tjurin variety is, up to isogeny, the Jacobi variety of the classical spectral curve. We sometimes drop the subscript on $\operatorname{Tur}_{\lambda} Y$ when it is only identified up to isogeny.

In preparation we develop some group theoretic material for the Weyl groups. We shall decompose the induced representations ind ${ }_{S_{\lambda}}^{W} 1$ and then describe the Hecke rings $H\left(S_{\lambda}, W\right)$ for $S_{\lambda}$ the stabilizer of the highest weight of the classical representation. Recall $[\mathrm{CR}]$

$$
\operatorname{ind}_{S}^{W} 1=\mathbf{C}[W] \otimes_{\mathbf{C}[S]} 1 \cong \mathbf{C}[W / S] .
$$

For the various Lie algebras we use $\mathfrak{h}$ to denote the representation of $W$ on the Cartan subalgebra. For types other than $A_{n}$ there is a natural map $W \rightarrow \Sigma_{n}$. We use $\mathfrak{h}_{A_{n-1}}$ to denote the representation $W$ obtained by composing this map with the representation of $\Sigma_{n}$ on the type $A_{n-1}$ Cartan subalgebra. For the algebra of type $G_{2}$ the Weyl group is $\mathbf{Z}_{2} \times \Sigma_{3}$. Let $\chi_{(-1,1)}$ and $\chi_{(-1,-1)}$ be the 1-dimensional representations whose characters are $\chi_{(-1,1)}\left(t^{\epsilon} \sigma\right)=(-1)^{\epsilon}$ and $\chi_{(-1,-1)}\left(t^{\epsilon} \sigma\right)=$ $(-1)^{\epsilon} \operatorname{det} \sigma$.

Proposition 20. For algebras of type $A, B, C$, and $D$, let $S=S_{\lambda}$ be the stabilizer of the highest weight of the classical representation. For type $G_{2}$, let $\lambda_{1}=(0,-1,1)$ and $\lambda_{2}=(-1,-1,2)$. Then $S_{\lambda_{1}}=\langle t(2,3)\rangle$ and $S_{\lambda_{2}}=\langle(1,2)\rangle$. We have the following decompositions of $\operatorname{ind}_{S}^{W}=\mathbf{C}[W / S]$ into irreducible representations.

$$
\begin{array}{rlrl}
\text { type } A_{n} & \mathbf{C}[W / S] & =\mathfrak{h} \oplus 1 \\
\text { type } B_{n}, C_{n}, D_{n} & \mathbf{C}[W / S] & =\mathfrak{h} \oplus \mathfrak{h}_{A_{n-1}} \oplus 1 \\
\text { type } G_{2} & \mathbf{C}\left[W / S_{\lambda_{1}}\right] & =\mathfrak{h} \oplus \chi_{(-1,-1)} \oplus \mathfrak{h}_{A_{2}} \oplus 1 \\
\text { type } G_{2} & \mathbf{C}\left[W / S_{\lambda_{2}}\right] & =\mathfrak{h} \oplus \chi_{(-1,1)} \oplus \mathfrak{h}_{A_{2}} \oplus 1
\end{array}
$$

Proof. We do only the $\lambda_{1}$ case of $G_{2}$. The other cases are easier. 
Each left coset of $\langle t(2,3)\rangle$ contains a unique element of $\Sigma_{3}$ which is used to specify the coset, e.g., $(1,2,3) S_{\lambda_{1}}=C_{123}$. The representation $\mathbf{C}\left[W / S_{\lambda_{1}}\right]$ decomposes into four irreducible representations. $\Sigma_{3}$ permutes the set $\left\{C_{e}+C_{23}, C_{12}+C_{123}, C_{13}+\right.$ $\left.C_{132}\right\}$ and $\mathbf{Z}_{2}$ acts trivially. So the span of these 3 elements decomposes into $\mathfrak{h}_{A_{2}} \oplus$ trivial. The complement consists of two irreducible representations. The span of $C_{e}-C_{23}+C_{123}-C_{12}+C_{132}-C_{13}$ is the representation on which $\mathbf{Z}_{2}$ acts by -1 and $\Sigma_{3}$ acts as multiplication by the sign of the permutation. This representation is $\chi_{(-1,-1)}$. The last irreducible summand is contained in the span of $e_{1}=C_{123}-C_{12}-C_{132}+C_{13}, e_{2}=C_{132}-C_{13}-C_{e}+C_{23}$, and $e_{3}=C_{e}-C_{23}-$ $C_{123}+C_{12}$. It is $\left\{a e_{1}+b e_{2}+c e_{3} \mid a+b+c=0\right\}$ which is isomorphic to $\mathfrak{h}_{G_{2}}$. So

$$
\mathbf{C}\left[W / S_{\lambda_{1}}\right]=\mathfrak{h} \oplus \chi_{(-1,-1)} \oplus \mathfrak{h}_{A_{2}} \oplus \text { trivial. }
$$

We note that if $\Sigma_{\text {sk }}=\left\{t^{\operatorname{sign}(\sigma)} \sigma \mid \sigma \in \Sigma_{3}\right\}$ then $\operatorname{ind}_{\Sigma_{\text {sk }}}^{W} 1=\chi_{(-1,-1)} \oplus 1$. Also $\operatorname{ind}_{\mathbf{Z}_{2} \times \Sigma_{2}}^{W} 1=\mathfrak{h}_{A_{2}} \oplus$ trivial, so the above decomposition may be produced by inducing up through the subgroups $\Sigma_{\text {sk }}$ and $\mathbf{Z}_{2} \times \Sigma_{2}$. In addition the decomposition of $\mathbf{C}\left[W / S_{\lambda_{2}}\right]$ could also be written using $\chi_{(-1,1)} \oplus 1=\operatorname{ind}_{\Sigma_{3}}^{\mathbf{Z}_{2} \times \Sigma_{3}} 1$.

We now examine the Hecke rings $H\left(S_{\lambda}, W\right)$ for $\lambda$ the highest weight of the classical representation for the algebras of type $B, C$, or $D$, and for the two weights $\lambda_{1}$ and $\lambda_{2}$ of $G_{2}$. In particular we identify explicitly the element $P_{\lambda}^{\lambda}$ used to define the Prym-Tjurin varieties.

For the rest of the section we abbreviate $S_{\lambda}$ by $S$ when the weight $\lambda$ is clear from context. The elements of the Hecke ring $H(S, W)$ are the double cosets of $S$ in $W$. In order to multiply two double cosets you write each double coset as a union or sum of right cosets, multiply out the right cosets by the formula $(S g)(S h)=S g h$, and reassemble each appropriate combination of right cosets into the double coset which is their union. So we will determine the double cosets and their decomposition into right cosets for the specific Hecke rings of interest.

Proposition 21. Let $\lambda$ be the highest weight of the classical representation for type $B$ or $C$. The Hecke ring $H\left(S_{\lambda}, W\right)$ is the free $\mathbf{Z}$-module $\mathbf{Z}\left[S e S, S t_{1} S, S(1,2) S\right]$. It is a commutative ring with unit $S e S$ and $S t_{1} S \cdot S(1,2) S=S(1,2) S$. Furthermore, $P_{\lambda}^{\lambda}=|S|\left(S e S-S t_{1} S\right)$.

Proof. $W=\mathbf{Z}_{2}^{n} \rtimes \Sigma_{n}$ and $S_{\lambda}=\mathbf{Z}_{2}^{n-1} \rtimes \Sigma_{n-1}$. In the $e_{i}$ basis for $\mathfrak{h}^{*}$, the highest weight of the classical representation is $\lambda=(1,0, \ldots, 0)$ for both type $B$ and type $C$. There are 3 double cosets of $S=S_{\lambda}$. They, and their decompositions into right cosets, are below.

$$
\begin{aligned}
S e S & =S e=\{\sigma \mid \sigma(1)=1\}, \\
S t_{1} S & =S t_{1}=\{\sigma \mid \sigma(-1)=1\}, \\
S(1,2) S & =\left(\bigcup_{k=2}^{n} S(1, k)\right) \bigcup\left(\bigcup_{k=2}^{n} S t_{1}(1, k)\right)=\left\{\sigma \mid \sigma^{-1}(1) \neq \pm 1\right\} .
\end{aligned}
$$

The coset $S e S$ serves as a multiplicative identity in $H(S, W)$. By direct calculation $(S(1,2) S)\left(S t_{1} S\right)=\sum_{k=2}^{n} S(1, k) t_{1}+\sum_{k=2}^{n} S t_{1}(1, k) t_{1}$ which consists of $2 n-$ 2 right cosets all contained in $S(1,2) S$. So $(S(1,2) S)\left(S t_{1} S\right)=S(1,2) S$ and a similar calculation shows $\left(S t_{1} S\right)(S(1,2) S)=S(1,2) S$. We observe that $H(S, W)$ is commutative. 
We now identify the element $P_{\lambda}^{\lambda} \in H(S, W)$. From the definition,

$$
P_{\lambda}^{\lambda}=|S| \sum_{w: S \backslash W / S}\left\langle w^{-1}(\lambda), \lambda\right\rangle S w S=|S|\left(S e S-S t_{1} S\right) .
$$

Proposition 22. Let $\lambda$ be the highest weight of the classical representation for type $D$. The Hecke ring $H\left(S_{\lambda}, W\right)$ is the free $\mathbf{Z}$-module $\mathbf{Z}\left[S e S, S t_{1} t_{2} S, S(1,2) S\right]$. It is a commutative ring with unit $S e S$ and $S t_{1} t_{2} S \cdot S(1,2) S=S(1,2) S$. Furthermore, $P_{\lambda}^{\lambda}=|S|\left(S e S-S t_{1} t_{2} S\right)$.

Proof. This case is almost identical to the previous case. $W=\mathbf{Z}_{2}^{n-1} \rtimes \Sigma_{n}$ and $S_{\lambda}=\mathbf{Z}_{2}^{n-2} \rtimes \Sigma_{n-1}$. In the $e_{i}$ basis for $\mathfrak{h}^{*}$, the highest weight of the classical representation is $\lambda=(1,0, \ldots, 0)$. The double cosets and their decomposition as a union of right cosets are below.

$$
\begin{aligned}
S e S & =S e \\
S t_{1} t_{2} S & =S t_{1} t_{2}, \\
S(1,2) S & =\left(\bigcup_{k=2}^{n} S(1, k)\right) \cup\left(\bigcup_{k=2}^{n} t_{1} t_{2} S(1, k)\right) .
\end{aligned}
$$

As in the previous case the Hecke ring is commutative. In fact the obvious set isomorphism of the three cosets that generate the Hecke rings for the two cases extends to an isomorphism of the Hecke rings. Moreover, for the $D_{n}$ case we also get $P_{\lambda}^{\lambda}=|S|\left(S e S-S t_{1} t_{2} S\right)$.

Proposition 23. Let $\lambda_{1}=(0,-1,1)$ be the first fundamental weight for type $G_{2}$. The Hecke ring $H\left(S_{\lambda_{1}}, W\right)$ is the free $\mathbf{Z}$-module $\mathbf{Z}[S e S, S t S, S(1,2) S, S t(1,2) S]$. It is a commutative ring with unit $S e S$. Furthermore,

$$
P_{\lambda_{1}}^{\lambda_{1}}=2[S e S-S t S][2 S e S-S t(1,2) S] .
$$

Let $\lambda_{2}=(-1,-1,2)$ be the second fundamental weight for type $G_{2}$. The Hecke ring $H\left(S_{\lambda_{2}}, W\right)$ is the free $\mathbf{Z}$-module $\mathbf{Z}[S e S, S t S, S(1,2) S, S t(1,2) S]$ with now $S=$ $S_{\lambda_{2}}$. It is a commutative ring with unit $S e S$. Furthermore,

$$
P_{\lambda_{2}}^{\lambda_{2}}=2[S e S-S t S][2 S e S-S t(1,2) S] .
$$

Proof. For type $G_{2}, W=\mathbf{Z}_{2} \times \Sigma_{3}$ and $S_{\lambda_{1}}=\langle t(2,3)\rangle$. There are four double cosets, namely,

$$
\begin{aligned}
S e S & =\{e, t(2,3)\}=S e, \\
S t S & =\{t,(2,3)\}=S t, \\
S(1,2) S & =\{(1,2),(1,3), t(1,2,3), t(1,3,2)\}=S(1,2) \cup S(1,3), \\
S t(1,2) S & =\{t(1,2), t(1,3),(1,2,3),(1,3,2)\}=S t(1,2) \cup S t(1,3) .
\end{aligned}
$$

Again $H(S, W)$ is a commutative Hecke algebra. If we write $\epsilon=S e S, \tau=S t S$, $\sigma=S(1,2) S$, and $\omega=S t(1,2) S$, then the multiplication table is $\tau^{2}=\epsilon, \sigma^{2}=\omega^{2}=$ $2 \epsilon+\omega, \sigma \omega=2 \tau+\sigma, \tau \sigma=\omega$, and $\tau \omega=\sigma$.

Direct computation of the element $P_{\lambda_{1}}^{\lambda_{1}}=|S| \sum_{w: S \backslash W / S}\left\langle w^{-1}\left(\lambda_{1}\right), \lambda_{1}\right\rangle S w S$ gives

$$
\begin{aligned}
P_{\lambda_{1}}^{\lambda_{1}} & =2[2 S e S-2(S t S)+S(1,2) S-(S t(1,2) S)] \\
& =2[S e S-(S t S)][2 S e S-S t(1,2) S] .
\end{aligned}
$$


For $S=S_{\lambda_{2}}=\langle(1,2)\rangle$, there are again four double cosets, namely, $\epsilon=S e S$, $\tau=S t S, \sigma=S t(1,2) S$, and $\omega=S(1,2) S$. Let $\phi$ be the outer automorphism of $W$ given by $\phi: t^{\epsilon} \sigma \mapsto t^{\epsilon+\operatorname{sign}(\sigma)} \sigma$, for $\sigma \in \Sigma_{3}$. The automorphism of $W$ that consists of $\phi$ followed by conjugation by $(1,3)$ maps $S_{\lambda_{1}}$ to $S_{\lambda_{2}}$ and induces an isomorphism of Hecke rings. The element $P_{\lambda_{2}}^{\lambda_{2}}$ is computed as in the previous example.

Now we can show that the Prym-Tjurin varieties for types $B, C$, and $D$ are Pryms. Let $Y_{1}$ be the classical spectral curve. Let $Y_{A}$ be the quotient curve $Y_{A}=Y /\left(\mathbf{Z}_{2}^{n} \rtimes \Sigma_{n-1}\right)$ for types $B_{n}$ and $C_{n}$ and $Y_{A}=Y /\left(\mathbf{Z}_{2}^{n-1} \rtimes \Sigma_{n-1}\right)$ for type $D_{n}$. There is a double covering $Y_{1} \rightarrow Y_{A}$ in each case. Write $S$ for the stabilizer $S_{\lambda}$ of the highest weight $\lambda$ of the classical representation. We write 1 for the identity element in $H(S, W)$.

Lemma 24. The involution that interchanges the sheets of the double covering $Y_{1} \rightarrow Y_{A}$ is given by the correspondence $S t_{1} S$ for algebras of type $B$ or $C$ and by $S t_{1} t_{2} S$ for algebras of type $D$.

Proof. Let the Weyl group element $t$ be $t=t_{1}$ for types $B$ or $C$ and $t=t_{1} t_{2}$ for type $D$. Let $x \in Y$. Then $x$ projects to $S x \in Y_{1}$. We observe the action of $a=S t S=S t$ on the point $S x . C_{a}(S x)=\pi_{\lambda}(\bar{a} \cdot x)$ where $\bar{a}=t$. So $C_{a}(S x)=\pi_{\lambda}(t x)=(S t) \cdot x$. Now $S t \neq S$ and $S t \cup S=A$. Hence $C_{a}$ flips the sheets of the double cover $Y_{1} \rightarrow Y_{A}$.

Theorem 25. $\operatorname{Tur}_{\lambda}\left(Y_{1}\right)$ is the Prym variety of the double covering $Y_{1} \rightarrow Y_{A}$ for the algebras of type $B, C$, or $D$.

Proof. The Prym is the image of $(1-S t S)$ in $\operatorname{Jac}_{1}$ while $\operatorname{Tur}_{\lambda}\left(Y_{1}\right)$ is the image of $P_{\lambda}$ in Jac $Y_{1}$. But we have previously shown that $P_{\lambda}=|S|(1-S t S)$ so the two maps have the same image.

The Prym-Tjurin varieties for type $G_{2}$ cannot in general be expected to be Pryms. Two non-parabolic subgroups lie between $S_{\lambda_{1}}=\langle t(2,3)\rangle$ and $W=\mathbf{Z}_{2} \times \Sigma_{3}$, namely $\mathbf{Z}_{2} \times \Sigma_{2}=\langle t,(2,3)\rangle$ and $\Sigma_{\text {sk }}=\left\{t^{\operatorname{sign}(\sigma)} \sigma \mid \sigma \in \Sigma_{3}\right\}$, a "skewed "copy of $\Sigma_{3}$ inside $W$.

We lay out notation for the $G_{2}$ curves and the projections between them. For $i=1$ or 2 , we write $Y_{i}=Y_{\lambda_{i}}=Y / S_{\lambda_{i}}$ for the spectral curves. We write $Y_{A_{i}}$ or just $Y_{A}$ for $Y /\left(\mathbf{Z}_{2} \times \Sigma_{2}\right)$ where the $\Sigma_{2}$ factor is generated by $(2,3)$ for $\lambda_{1}$ and by $(1,2)$ for $\lambda_{2}$. . Write $\pi_{A}$ for the double covering map $\pi_{A}: Y_{i} \rightarrow Y_{A}$. We also write $Y_{\mathrm{sk}}=Y / \Sigma_{\mathrm{sk}}$ and $\pi_{\mathrm{sk}}$ for the triple covering $\pi_{\mathrm{sk}}: Y_{1} \rightarrow Y_{\mathrm{sk}}$. Similarly $Y_{\Sigma}=Y / \Sigma_{3}$ and $\pi_{\Sigma}: Y_{2} \rightarrow Y_{\Sigma}$ is the other triple covering. We abbreviate $S_{\lambda_{i}}$ by $S_{\lambda}$ or just $S$ when there is no danger of confusion. Finally, we let elements of the Hecke algebra $H\left(S_{\lambda_{i}}, W\right)$ also designate the associated correspondence on $Y_{i}$ and endomorphism of $\operatorname{Jac} Y_{i}$.

The following proposition gives the meaning of the factorization $P_{\lambda_{i}}^{\lambda_{i}}=$ $2[1-S t S][2-S t(1,2) S]$.

\section{Proposition 26.}

(1) For either $i=1$ or 2, the image $\pi_{A}^{*}\left(\operatorname{Jac} Y_{A}\right)$ is the identity component of $\operatorname{ker}(1-S t S)$ in $\operatorname{Jac} Y_{i}$. The image of $1-S t S$ is the Prym variety of the double cover $Y_{i} \rightarrow Y_{A}$. Each Prym-Tjurin is contained in the Prym variety of the respective double cover. 
(2) $\pi_{\mathrm{sk}}^{*}\left(\mathrm{Jac} Y_{\mathrm{sk}}\right)$ is contained in $\operatorname{ker}[2-S t(1,2) S] \subset \operatorname{Jac} Y_{1}$. The Prym-Tjurin variety $\operatorname{Tur}_{\lambda_{1}}\left(Y_{\lambda_{1}}\right)$ is transverse to $\pi_{\mathrm{sk}}^{*}\left(\mathrm{Jac} Y_{\mathrm{sk}}\right)$. Similarly $\pi_{\Sigma}^{*}\left(\mathrm{Jac} Y_{\Sigma}\right)$ is contained in $\operatorname{ker}[2-S t(1,2) S] \subset \operatorname{Jac} Y_{2}$ and $\operatorname{Tur}_{\lambda_{2}}\left(Y_{\lambda_{2}}\right)$ is transverse to $\pi_{\Sigma}^{*}\left(\operatorname{Jac} Y_{\Sigma}\right)$.

Proof. We present only the $\lambda=\lambda_{1}$ case as the other case is completely similar.

(1) Just as for type $B$, we check that the correspondence $S t S$ interchanges the sheets of the cover $Y_{1} \rightarrow Y_{A}$, If $x_{1} \in Y_{1 *}$ and $x \in Y_{*}$ is a preimage of $x_{1}$ under $\pi_{\lambda}: Y \rightarrow Y_{1}$, then $(S t S) \cdot x_{1}=\pi_{\lambda}(t \cdot x)$. Since $t \notin S=\langle t(2,3)\rangle$, then $\pi_{\lambda}(t \cdot x) \neq x_{1}$. So $S t S$ does interchange the sheets of the cover. Consequently the Prym variety of the double cover $Y_{\lambda} \rightarrow Y_{A}$ is the image of $(1-S t S)$ in $\operatorname{Jac} Y_{1}$. Now, $\operatorname{Tur}_{\lambda}\left(Y_{1}\right)$ is the image of $P_{\lambda}^{\lambda}$ in $\operatorname{Jac} Y_{1}$. We have previously shown that $P_{\lambda}=2(1-S t S)(2-S t(1,2) S)$ so the image of $P_{\lambda}^{\lambda}$ is contained in the image of $(1-S t S)$.

(2) We check that if $x_{\mathrm{sk}} \in Y / \Sigma_{\mathrm{sk}}$ then the correspondence $2-S t(1,2) S$ maps $\pi_{\mathrm{sk}}\left(x_{\mathrm{sk}}\right)$ to the zero divisor. First if $x_{1} \in Y_{1}$ and $x \in Y$ is such that $\pi_{\lambda}(x)=$ $x_{1}$ then

$(2-S t(1,2) S) \cdot x_{1}=\pi_{\lambda}((2-t(1,2)-t(1,3)) \cdot x)=3 x_{1}-\pi_{\mathrm{sk}}^{-1} \pi_{\mathrm{sk}}\left(x_{1}\right)$.

Therefore $(2-S t(1,2) S) \cdot \pi_{\mathrm{sk}}^{-1}\left(x_{\mathrm{sk}}\right)=3 \pi_{\mathrm{sk}}^{-1}\left(x_{\mathrm{sk}}\right)-\pi_{\mathrm{sk}}^{-1} \pi_{\mathrm{sk}} \pi_{\mathrm{sk}}^{-1}\left(x_{\mathrm{sk}}\right)=0$ since $\pi_{\mathrm{sk}} \circ \pi_{\mathrm{sk}}^{-1}$ is multiplication by 3 . Since $(2-\operatorname{St}(1,2) S)\left(\pi_{\mathrm{sk}}^{*}\left(\mathrm{Jac} Y_{\mathrm{sk}}\right)\right)=e \in$ $\operatorname{Jac} Y_{\lambda_{1}}$, the Prym-Tjurin is transverse to the image of Jac $Y_{\mathrm{sk}}$.

We now switch techniques from Hecke algebras to induced representations to consider type $A_{n}$ and to decompose Jac $Y_{\lambda_{i}}$ for type $G_{2}$. Proposition 29 will imply that $\pi_{\mathrm{sk}}^{*}\left(\mathrm{Jac} Y_{\mathrm{sk}}\right)$ is the identity component of $\operatorname{ker}(2-\operatorname{St}(1,2) S)$ in $\operatorname{Jac} Y_{1}$.

Theorem 27. Let $\mathfrak{g}$ be a Lie algebra of type $A_{n}$, i.e. $\operatorname{sl}(n+1, \mathbf{C})$. Let $Y_{1}$ denote the classical spectral curve. Then

$$
\operatorname{dim} \operatorname{Tur} Y=\operatorname{dim} \operatorname{Jac} Y_{1}-\frac{1}{2} \operatorname{dim} H^{1}(P) .
$$

In particular when $P$ is the Riemann sphere, Jac $Y_{1}$ equals Tur $Y$ up to isogeny.

Proof. $W=\Sigma_{n+1}, S_{\lambda}=\Sigma_{n}$ and $Y_{1}=Y / \Sigma_{n}$. From Proposition 20,

$$
\operatorname{ind}_{\Sigma_{n}}^{\Sigma_{n+1}} 1=\mathbf{C}\left[\Sigma_{n+1} / \Sigma_{n}\right]=\mathfrak{h} \oplus 1 .
$$

Now, as in the proof of Lemma 16, $\operatorname{dim} \operatorname{Tur} Y=\left\langle\mathfrak{h}, T_{e} \mathrm{Jac} Y\right\rangle$. We also have

$$
\begin{aligned}
\left\langle 1, T_{e} \mathrm{Jac} Y\right\rangle & =\operatorname{dim}\left(T_{e} \mathrm{Jac} Y\right)^{\Sigma_{n+1}} \\
& =\operatorname{dim} H^{(0,1)}(Y, \mathbf{C})^{\Sigma_{n+1}} \\
& =\frac{1}{2} \operatorname{dim} H^{1}(Y, \mathbf{C})^{\Sigma_{n+1}} \\
& =\frac{1}{2} \operatorname{dim} H^{1}(P, \mathbf{C}) .
\end{aligned}
$$


This last equality is obtained by the transfer homomorphism [B, pp. 37-38]. Consequently,

$$
\begin{aligned}
\frac{1}{2} \operatorname{dim} H^{1}(P, \mathbf{C})+\operatorname{dim} \operatorname{Tur} Y & =\left\langle 1+\mathfrak{h}, T_{e} \mathrm{Jac} Y\right\rangle \\
& =\left\langle\operatorname{ind}_{\Sigma_{n}}^{\Sigma_{n+1}} 1, T_{e} \mathrm{Jac} Y\right\rangle \\
& =\left\langle\operatorname{res}_{\Sigma_{n}}^{\Sigma_{n+1}} T_{e} \mathrm{Jac} Y, 1\right\rangle \quad \text { by Frobenius reciprocity } \\
& =\operatorname{dim}\left(T_{e} \mathrm{Jac} Y\right)^{\Sigma_{n}} \\
& =\operatorname{dim}\left(T_{e} \mathrm{Jac}\left(Y / \Sigma_{n}\right)\right)
\end{aligned}
$$

again by a transfer homomorphism argument.

The $W$ module $T_{e}$ Jac $Y$ decomposes into a direct sum of submodules according to $\chi$-types for irreducible representations $\chi$ of $W$. The decomposition can be passed down to the tangent spaces $T_{e} \operatorname{Jac}(Y / S)$ of the Jacobi varieties of the quotient curves for $S$ a subgroup of $W$. Let $\pi_{S}: Y \rightarrow Y / S$ be the projection and $d \pi_{S}^{*}$ the corresponding map $d \pi_{S}^{*}: T_{e} \mathrm{Jac}(Y / S) \rightarrow T_{e} \mathrm{Jac} Y$.

Define

$$
\left(T_{e} \operatorname{Jac}(Y / S)\right)_{\chi}=\left(d \pi_{S}^{*}\right)^{-1}\left[\left(T_{e} \operatorname{Jac} Y\right)_{\chi}\right]
$$

where $\left(d \pi_{S}^{*}\right)^{-1}$ means the inverse image. $\left(T_{e} \mathrm{Jac}(Y / S)\right)_{\chi}$ is a module over $H(S, W)$.

Lemma 28. Let $\chi^{S}$ and $\chi^{T}$ be the subsets of a representation space for $\chi$ fixed by the respective subgroups $S$ and $T$.

$$
T_{e} \mathrm{Jac} Y / S=\sum_{\left\langle\chi, \operatorname{ind}_{S}^{W} 1\right\rangle \neq 0}\left(T_{e} \mathrm{Jac} Y / S\right)_{\chi} .
$$

(2) If $S \subset T \subset W$ and $\pi_{T S}: Y / S \rightarrow Y / T$ is the projection then

$$
d \pi_{T S}^{*}\left(T_{e} \operatorname{Jac} Y / T\right)_{\chi} \subset\left(T_{e} \mathrm{Jac} Y / S\right)_{\chi} .
$$

(3) $\operatorname{dim}\left(T_{e} \operatorname{Jac} Y / S\right)_{\chi}=\left\langle T_{e} \operatorname{Jac} Y, \chi\right\rangle \operatorname{dim} \chi^{S}$.

(4) The map $d \pi_{T S}^{*}:\left(T_{e} \mathrm{Jac} Y / T\right)_{\chi} \rightarrow\left(T_{e} \mathrm{Jac} Y / S\right)_{\chi}$ is onto iff $\chi^{S}=\chi^{T}$.

Proof. We first show $T_{e} \mathrm{Jac} Y / S=\sum_{\chi}\left(T_{e} \mathrm{Jac} Y / S\right)_{\chi}$ where the direct sum runs over all irreducible representations of $W$. The map $d \pi_{S}^{*}: T_{e} \mathrm{Jac} Y / S \rightarrow T_{e} \mathrm{Jac} Y$ is oneto-one and its image is $\left(T_{e} \mathrm{Jac} Y\right)^{S}$, the subspace of $T_{e} \mathrm{Jac} Y$ of vectors fixed by $S$. In fact, viewing $T_{e} \mathrm{Jac} Y$ as $H^{(0,1)}(Y, \mathbf{C})$, this follows from the transfer argument since $H^{1}(Y, \mathbf{C})=H^{(0,1)}(Y) \oplus H^{(1,0)}(Y)$ as $W$ modules. So it is sufficient to show $d \pi_{S}^{*}\left(T_{e} \operatorname{Jac} Y / S\right)=d \pi_{S}^{*}\left(\sum_{\chi}\left(T_{e} \operatorname{Jac} Y / S\right)_{\chi}\right)$. Starting with the right-hand side we calculate

$$
\begin{aligned}
d \pi_{S}^{*}\left(\sum_{\chi}\left(T_{e} \operatorname{Jac} Y / S\right)_{\chi}\right) & =\sum_{\chi}\left(d \pi_{S}^{*}\left[\left(d \pi_{S}^{*}\right)^{-1}(\operatorname{Jac} Y)_{\chi}\right]\right) \\
& =\sum_{\chi}\left(d \pi_{S}^{*}\left(d \pi_{S}^{*}\right)^{-1}(\operatorname{Jac} Y)_{\chi}\right)^{S}
\end{aligned}
$$


since each $(\operatorname{Jac} Y)_{\chi}$ is invariant under $W$, hence $S$,

$$
\begin{aligned}
& =\sum_{\chi}\left[(\operatorname{Jac} Y)_{\chi}\right]^{S}=\left(T_{e} \operatorname{Jac} Y\right)^{S} \\
& =d \pi_{S}^{*}\left(T_{e} \operatorname{Jac} Y / S\right) .
\end{aligned}
$$

Before completing the proof of statement 1 we verify the other three statements. If $S \subset T \subset W$ then $d \pi_{T}^{*}=d \pi_{S}^{*} \circ d \pi_{T S}^{*}$. Since $d \pi_{T}^{*}\left(\left(T_{e} \operatorname{Jac} Y / T\right)_{\chi}\right) \subset\left(T_{e} \mathrm{Jac} Y\right)_{\chi}$ we have $d \pi_{T S}^{*}\left(\left(T_{e} \operatorname{Jac} Y / T\right)_{\chi}\right) \subset\left(d \pi_{S}^{*}\right)^{-1}\left(T_{e} \mathrm{Jac} Y\right)_{\chi}=\left(T_{e} \mathrm{Jac} Y / S\right)_{\chi}$, which is statement 2. Statement 3 follows from $d \pi_{S}^{*}: T_{e} \mathrm{Jac} Y / S \rightarrow\left(T_{e} \mathrm{Jac} Y\right)^{S}$ being an isomorphism. Statement 4 is an immediate consequence of statements 2 and 3 . Finally we complete statement 1 . If $\left\langle\operatorname{ind}_{S}^{W} 1, \chi\right\rangle=0$ then, by Frobenius reciprocity, $\left\langle\operatorname{res}_{S}^{W} \chi, 1\right\rangle=0$ or $\operatorname{dim} \chi^{S}=0$. Hence only those irreducibles $\chi$ which occur in $\operatorname{ind}_{S}^{W} 1$ appear in the decomposition $T_{e} \mathrm{Jac} Y / S=\sum_{\chi}\left(T_{e} \mathrm{Jac} Y / S\right)_{\chi}$ as Hecke modules.

We resume the previous notation for the $G_{2}$ quotient curves and projections between them. The following proposition gives more detail on the Jacobi varieties for the $G_{2}$ spectral curves.

Proposition 29. For the Lie algebra of type $G_{2}$, up to isogeny

$$
\operatorname{Jac} Y / S_{\lambda_{1}}=\operatorname{Tur}_{\lambda_{1}}\left(Y_{\lambda_{1}}\right) \oplus\left(\pi_{A_{1}}^{*} \operatorname{Jac} Y_{A_{1}}+\pi_{\mathrm{sk}}^{*} \operatorname{Jac} Y_{\mathrm{sk}}\right)
$$

and

$$
\operatorname{Jac} Y / S_{\lambda_{2}}=\operatorname{Tur}_{\lambda_{2}}\left(Y_{\lambda_{2}}\right) \oplus\left(\pi_{A_{2}}^{*} \operatorname{Jac} Y_{A_{2}}+\pi_{\Sigma}^{*} \operatorname{Jac} Y_{\Sigma}\right) .
$$

If $P$ is the Riemann sphere then the sums are direct up to isogeny.

Proof. We discuss only the case of $\lambda_{1}$ as the $\lambda_{2}$ case is entirely similar. By Proposition 20, $\operatorname{ind}_{S_{\lambda_{1}}}^{W} 1=\mathfrak{h} \oplus \chi_{(-1,-1)} \oplus \mathfrak{h}_{A_{2}} \oplus 1$. The definition above Lemma 28 and the Proposition 15 together imply that $T_{e} \operatorname{Tur}_{\lambda_{1}}\left(Y_{\lambda_{1}}\right)$ is contained in $\left(T_{e} \operatorname{Jac} Y / S_{\lambda_{1}}\right)_{\mathfrak{h}}$. Since $\operatorname{dim} \mathfrak{h}^{S_{\lambda_{1}}}=1, T_{e} \operatorname{Tur}_{\lambda_{1}}\left(Y_{\lambda_{1}}\right)=\left(T_{e} \operatorname{Jac} Y / S_{\lambda_{1}}\right)_{\mathfrak{h}}$. We use statement 3 of Lemma 28 to check that $d \pi_{\mathrm{sk}}^{*}$ maps $T_{e}$ Jac $Y / \Sigma_{\mathrm{sk}}$ onto the the $\chi_{(-1,-1)}$ summand of $T_{e} \mathrm{Jac} Y / S_{\lambda_{1}}$. This follows from $\operatorname{dim}\left(\chi_{(-1,-1)}\right)^{S_{\lambda_{1}}}=1=\operatorname{dim}\left(\chi_{(-1,-1)}\right)^{\Sigma_{\mathrm{sk}}}$ as previously revealed from the decompositions of induced representations. The same sort of check shows that $T_{e} \mathrm{Jac} Y /\left(\mathbf{Z} \times \Sigma_{2}\right)$ maps onto the $\mathfrak{h}_{A_{2}}$ summand of $T_{e} \mathrm{Jac} Y / S_{\lambda_{1}}$. Finally both $T_{e} \operatorname{Jac} Y / \Sigma_{\text {sk }}$ and $T_{e} \operatorname{Jac} Y /\left(\mathbf{Z} \times \Sigma_{2}\right)$ and indeed $T_{e}$ Jac $P$ map onto the summand of $T_{e} \mathrm{Jac} Y / S_{\lambda_{1}}$ corresponding to the trivial representation.

Theorem 30. For the Lie algebra of type $G_{2}$ neither of the Prym-Tjurin varieties $\operatorname{Tur}_{\lambda_{i}}\left(Y_{\lambda_{i}}\right)$ is in general the Prym variety of $Y_{i} \rightarrow Y_{A_{i}}$.

Proof. It follows from the previous proposition that $\operatorname{Tur}_{\lambda_{1}}\left(Y_{\lambda_{1}}\right)$ will be the Prym variety of $Y_{1} \rightarrow Y_{A_{1}}$ only if $Y / \Sigma_{\text {sk }}$ has the same genus as the parameter space. Similarly $\operatorname{Tur}_{\lambda_{2}}\left(Y_{\lambda_{2}}\right)$ will be the Prym variety of $Y_{2} \rightarrow Y_{A_{1}}$ only if $Y / \Sigma_{3}$ has the same genus as the parameter space. In the section the Periodic Toda lattice we shall see that the periodic Toda lattice of type $G_{2}$ provides a counterexample in both cases.

For type $G_{2}$ the property that the Prym-Tjurin is a Prym consistent with the group theory could be destroyed by a change of base for the hierarchy of covering spaces. For example, suppose both $P$ and $Y / \Sigma_{\text {sk }}$ are Riemann spheres. The parameter space $P$ may be replaced via a ramified cover $Q \rightarrow P$ giving a new map 
$A: Q \rightarrow \mathfrak{g}$ by composition. Write $Z$ in place of $Y$ to denote spectral curves for the system parameterized by $Q$. If $Q$ is an elliptic curve which is a double cover of $P$ then $Z / \Sigma_{\text {sk }}$ is a curve of genus 3 . Then $\operatorname{Tur}_{\lambda_{1}}\left(Z_{\lambda_{1}}\right)$ is not the Prym variety of $Z_{1} \rightarrow Z_{A_{1}}$; neither, by the same sort of reasoning, is $\operatorname{Tur}_{\lambda_{2}}\left(Z_{\lambda_{2}}\right)$ the Prym variety of $Z_{2} \rightarrow Z_{A_{2}}$.

\section{Periodic Toda lattice}

In this section we study the periodic Toda lattices. Theorem 13 says that the Prym-Tjurin varieties $\operatorname{Tur}_{\lambda}\left(Y_{\gamma}\right)$ are all isogenous. In Corollary 34 we give a mild criterion under which for a fixed weight $\lambda$ the Prym-Tjurin varieties $\operatorname{Tur}_{\lambda}\left(Y_{\gamma}\right)$ are isomorphic. This criterion is satisfied by the periodic Toda lattices for generic initial conditions. The dimension formula of Theorem 17 is then applied to the periodic Toda lattices. The formula is evaluated with the help of the monodromy representation. The $\operatorname{sl}(4, \mathbf{C})$ Toda lattice is examined in detail.

For each simple Lie algebra there is a system of periodic Toda lattice equations [AvM1], [Mc2], [OP]. These equations can be expressed as a Lax equation $\frac{d A}{d t}=$ $[A, B]$ where $A(t)$ and $B(t)$ take values in the associated Lie algebra and depend on a spectral parameter. The parameter space is the Riemann sphere. For each initial condition there is a hierarchy of spectral curves and covering maps. For each algebra there is a Zariski open subset of phase space, i.e., a set of generic initial conditions, such that the topological structure of the ramified covers depends only on the underlying Lie algebra and not on the particular initial condition. The structures for two generic initial conditions $A_{1}: P \rightarrow \mathfrak{g}$ and $A_{2}: P \rightarrow \mathfrak{g}$ agree in the sense that there exist homeomorphisms $\phi$ and $\bar{\phi}$ that make the diagram

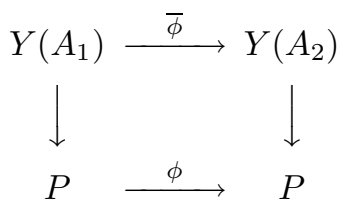

commute, where $Y\left(A_{i}\right)$ are the respective master curves. Our criterion in Corollary 34 and then the dimension formula will be used in this generic situation.

Generally throughout this section we will assume that the map $\mu: \pi_{1}\left(P_{*}\right) \rightarrow W$ is onto so that all the spectral curves $Y_{\lambda}$ are connected. Results can be modified to hold without this assumption.

Suppose $\gamma, \lambda$, and $\theta$ are weights with nested stabilizers $S_{\gamma} \subset S_{\lambda} \subset S_{\theta}$. The covering $\pi_{\lambda \gamma}: Y_{\gamma} \rightarrow Y_{\lambda}$ of the spectral curves yields a map $\pi_{\lambda \gamma}^{*}: \operatorname{Jac} Y_{\lambda} \rightarrow \operatorname{Jac} Y_{\gamma}$ with a finite kernel which, moreover, maps $\operatorname{Tur}_{\theta}\left(Y_{\lambda}\right)$ onto $\operatorname{Tur}_{\theta}\left(Y_{\gamma}\right)$. The kernel of $\pi_{\lambda \gamma}^{*}: \operatorname{Jac} Y_{\lambda} \rightarrow \operatorname{Jac} Y_{\gamma}$ has been identified in Proposition 3 as the group of deck transformations of the maximal unramified abelian cover $Y_{U A}$ (not necessarily a spectral curve) between $Y_{\lambda}$ and $Y_{\gamma}$, i.e. $Y_{\gamma} \rightarrow Y_{U A} \rightarrow Y_{\lambda}$.

Any covering map $\pi_{\lambda \gamma}$ is a factor of the covering map $\pi_{\lambda}: Y \rightarrow Y_{\lambda}$, namely $\pi_{\lambda}=\pi_{\lambda \gamma} \pi_{\gamma}$. Therefore in order to show that $\pi_{\lambda \gamma}^{*}: \operatorname{Tur}_{\theta}\left(Y_{\lambda}\right) \rightarrow \operatorname{Tur}_{\theta}\left(Y_{\gamma}\right)$ is an isomorphism it is sufficient to show $\pi_{\lambda}^{*}$ has a trivial kernel. The plan is to show, for $S_{\lambda}$ a parabolic subgroup of $W$, that if $Y \rightarrow Y_{U A} \rightarrow Y_{\lambda}$ is such that $Y_{U A} \rightarrow Y_{\lambda}$ is an unramified abelian cover then $Y_{U A}=Y_{\lambda}$. To this end the next lemma gives a criterion that $Y_{U A} \rightarrow Y_{\lambda}$ must satisfy to be unramified.

The regular covering $Y_{*} \rightarrow P_{*}$ has the entire Weyl group as the group of deck transformations. For $Y_{\lambda}=Y / S_{\lambda}$ the covering $Y_{*} \rightarrow Y_{\lambda *}$ has $S_{\lambda}$ as its group of deck 
transformations. Let $Y_{A} \rightarrow Y_{\lambda}$ be an abelian extension. Write $N$ for the group of deck transformations of $Y \rightarrow Y_{A}$. Then $N \triangleleft S_{\lambda}$ is a normal subgroup such that the quotient group $A=S_{\lambda} / N$ is abelian.

We need terminology for a certain type of loop. Let $R \rightarrow Z$ be a ramified cover and $z \in Z$. Usually $z$ will be a ramification point. Let $D_{z}$ be a small open disk neighborhood of $z$ that includes no other ramification points other than $z$. Let $\beta$ be a loop that follows a path from the basepoint to $D_{z}$, winds once about $z$ within $D_{z}$, and returns back along the same path from $D_{z}$ to the basepoint. We shall refer to such a loop as a small loop about $z$. We use $\beta$ to denote small loops about points. If $Z_{*}$ is the complement of the set of ramification points, then the set of small loops about a particular point form a conjugacy class in $\pi_{1}\left(Z_{*}\right)$.

The embedding $Y_{\lambda *} \rightarrow Y_{\lambda}$ gives a map of fundamental groups $\pi_{1}\left(Y_{\lambda *}\right) \rightarrow \pi_{1}\left(Y_{\lambda}\right)$. Let $K$ be the kernel, $K=\operatorname{ker}\left(\pi_{1}\left(Y_{\lambda *}\right) \rightarrow \pi_{1}\left(Y_{\lambda}\right)\right)$.If $\left\{y_{i}\right\}=Y_{\lambda} \backslash Y_{\lambda *}$ then $K$ is generated by the small loops $\beta_{i}$ about the $y_{i}$ 's. Let

$$
\nu: \pi_{1}\left(Y_{\lambda *}\right) \rightarrow \pi_{1}\left(Y_{\lambda *}\right) / \pi_{1}\left(Y_{A *}\right)=A
$$

be the defining map of the abelian cover $Y_{A *} \rightarrow Y_{\lambda *}$. Also let the composite

$$
\pi_{1}\left(Y_{\lambda *}\right) \rightarrow \pi_{1}\left(P_{*}\right) \stackrel{\mu}{\rightarrow} W
$$

be denoted $\mu_{\lambda}$.

Lemma 31. The following are equivalent.

(1) The abelian cover $Y_{A} \rightarrow Y_{\lambda}$ is unramified.

(2) $K \subset \operatorname{ker} \nu$.

(3) $\mu_{\lambda}(K) \subset N$.

Proof. $1 \Leftrightarrow 2$ Suppose $K \subset$ ker $\nu$. Let $\beta$ be a small loop about a possible ramification point $x$ of $Y_{A} \rightarrow Y_{\lambda}$. Since $\beta \in K \subset \operatorname{ker} \nu$, it and the disk it bounds both lift. This gives a point in the fiber over $x$ such that the projection map is not singular. Since ker $\nu$ is normal all of the conjugates of $\beta$ also lift. So $x$ is not a ramification point and the cover is unramified. Conversely, if $Y_{A} \rightarrow Y_{\lambda}$ is unramified then any null homotopic loop in $Y_{\lambda}$ lifts and so is in $\operatorname{ker} \nu$. Any small loop about a point is null homotopic. Hence $K \subset \operatorname{ker} \nu$.

$2 \Leftrightarrow 3$ Since $Y_{\lambda *}=Y_{*} / S_{\lambda}$, im $\mu_{\lambda} \subset S_{\lambda}$. The sequence $0 \rightarrow N \rightarrow S_{\lambda} \stackrel{\alpha}{\rightarrow} A \rightarrow 0$ is exact. The map $\nu$ is $\alpha \circ \mu_{\lambda}$ since $Y_{*} \rightarrow Y_{A *} \rightarrow Y_{\lambda *}$ is a composite of covers. So $y \in \operatorname{ker} \nu$ iff $\mu_{\lambda}(y) \in \operatorname{ker} \alpha=N$. (See Figure 1.)

The small loops about ramification points generate the kernel of the map $\pi_{1}\left(P_{*}\right)$ $\rightarrow \pi_{1}(P)$ induced by the inclusion $P_{*} \rightarrow P$. For any of the covers $\pi_{\lambda}: Y_{\lambda} \rightarrow P$ let $p$ be a ramification point and $D_{p}$ a small disk as previously described. Suppose the inverse image $\pi_{\lambda}^{-1}(p)$ is the set $\left\{x_{i}\right\}$ and $\pi_{\lambda}^{-1}\left(D_{p}\right)$ is a union of disks $D_{x_{i}}$. Each $D_{x_{i}} \rightarrow D_{p}$ is a cover ramified over $p$. Consequently if a lift of a small loop about a ramification point is closed it is a small loop about some $x_{i}$ and $D_{x_{i}} \rightarrow D_{p}$ is a trivial cover.

For the next lemma we drop the assumption that $\mu$ maps onto $W$ as it may be of more general use.

Lemma 32. Suppose the map $\mu: \pi_{1}\left(P_{*}\right) \rightarrow W$ has image $M$. Let $\beta$ be a small loop about a ramification point $p$ of the cover $Y_{\lambda} \rightarrow P$. Let $\mu(\beta)=b$. Then for any element $m \in M$ such that $m b m^{-1} \in S_{\lambda}$, one has $m_{b m}^{-1} \in \mu_{\lambda}(K)$. 

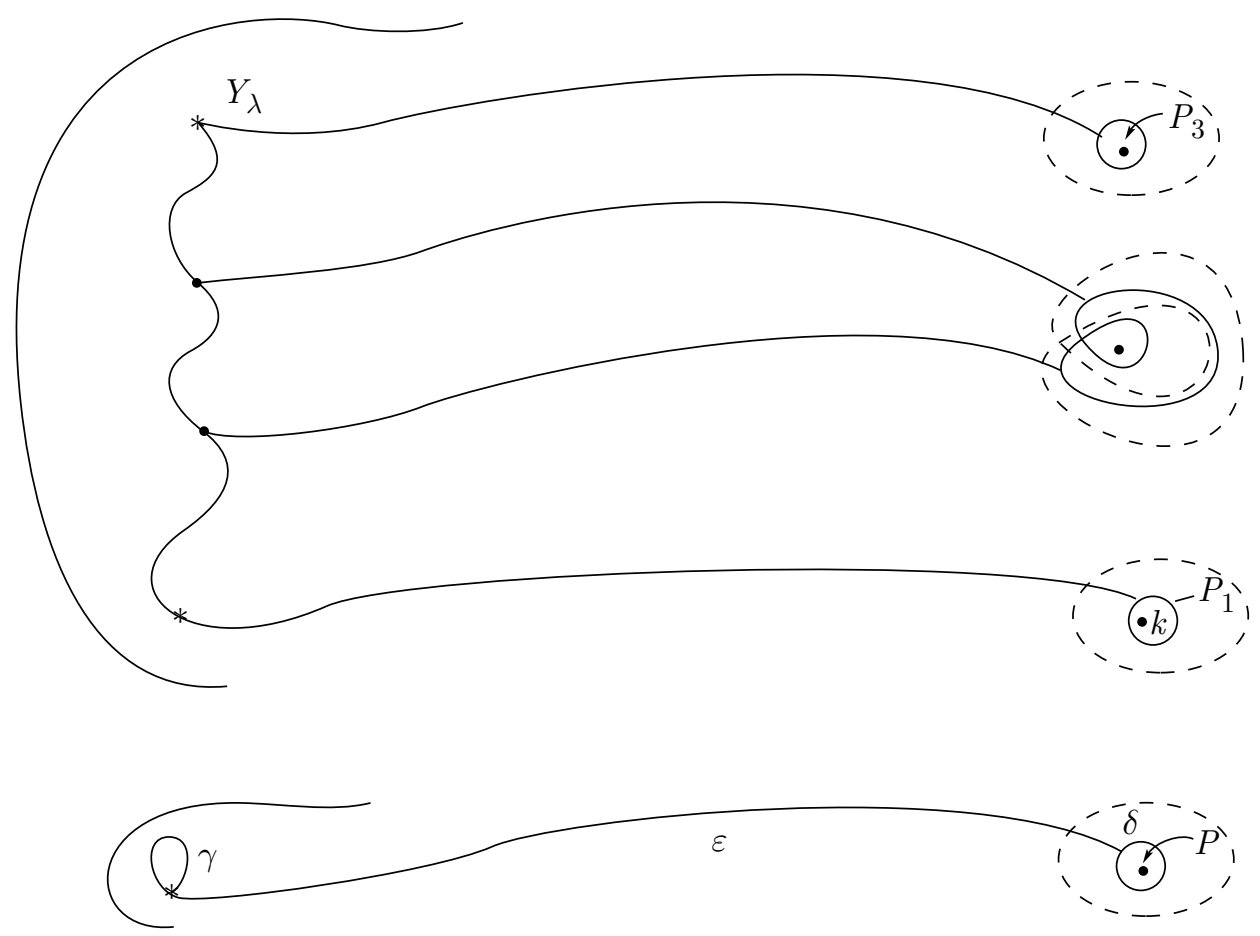

Figure 1. For the paths $\gamma, \epsilon$ and $\delta$ in $P$ the lifts of $\epsilon \delta \epsilon^{-1}$ and $\gamma^{3} \epsilon \delta \epsilon^{-1} \gamma^{-3}$ are small loops about $p_{1}$ and $p_{3}$ respectively.

Proof. Recall that the basepoints for the various spectral curves were chosen so that the projection maps are basepoint preserving. Let $X_{\lambda}$ be the connected component of $Y_{\lambda}$ containing the basepoint.

Choose a loop $\alpha$ representing an element of $\pi_{1}\left(P_{*}\right)$ such that $\mu(\alpha)=m$. Then the loop $\alpha \beta \alpha^{-1}$ obtained by following $\alpha$, then $\beta$, then returning by $\alpha^{-1}$ is also a small loop about $p$. Since $\mu\left(\alpha \beta \alpha^{-1}\right)=m b m^{-1} \in S_{\lambda}$ the loop $\alpha \beta \alpha^{-1}$ lifts. By the comments prior to the lemma, $m b m^{-1} \in \mu_{\lambda}(K)$.

Theorem 33. Suppose the map $\mu: \pi_{1}\left(P_{*}\right) \rightarrow W$ is onto $W$. Further suppose that $p_{i} \in P$ are ramification points for $i=1, \ldots, k$. Let $\beta_{i}$ be a small loop around $p_{i}$.

If $\left\{w \mu\left(\beta_{i}\right) w^{-1} \mid w \in W, 1 \leq i \leq k\right\} \cap S_{\lambda}$ generates $S_{\lambda}$ then $\pi_{\lambda}^{*}: \operatorname{Jac} Y_{\lambda} \rightarrow \operatorname{Jac} Y$ is injective, and moreover, the Prym-Tjurin varieties $\operatorname{Tur}_{\lambda}\left(Y_{\gamma}\right)$ are isomorphic for all $\gamma$ such that $S_{\gamma} \subset S_{\lambda}$.

Proof. By Lemma $32 w \mu\left(\beta_{i}\right) w^{-1} \in K$ so $\mu(K)=S_{\lambda}$. Then by Lemma $31 Y_{\lambda}$ has no unramified abelian covers that are covered by $Y$. By Proposition $3, \pi_{\lambda}^{*}$ is injective. Hence $\pi_{\lambda \gamma}^{*}$ is injective. By Theorem 13 the Prym-Tjurins are isomorphic.

We continue with the notation that $\beta_{1}$ and $\beta_{2}$ denote small loops about ramification points in $P_{*}$.

Corollary 34. Suppose $\mu$ is onto. The hypothesis of Theorem 33 is satisfied for all parabolics under any of the following conditions.

(1) $\mathfrak{g}$ has type $A_{n-1}$ and there exists $\beta_{1}$ such that $\mu\left(\beta_{1}\right)$ is a 2 -cycle in $\Sigma_{n}$, 
(2) $\mathfrak{g}$ has type $B_{n}$ or $C_{n}$ and there exist $\beta_{1}$ and $\beta_{2}$ such that $\mu\left(\beta_{1}\right)=t_{i}$ for some $i$ and either $\mu\left(\beta_{2}\right)=t_{j} t_{k}(j, k)$ or $\mu\left(\beta_{2}\right)=(j, k)$ for some $j \neq k$,

(3) $\mathfrak{g}$ has type $D_{n}$ and there exists $\beta_{1}$ such that either $\mu\left(\beta_{1}\right)$ is a 2-cycle in $\Sigma_{n} \subset \mathbf{Z}_{2}^{n-1} \rtimes \Sigma_{n}$ or alternatively $\mu\left(\beta_{1}\right)$ is an element of the form $t_{j} t_{k}(j, k)$.

In these cases, for each fixed weight $\lambda$, the Prym-Tjurin varieties $\operatorname{Tur}_{\lambda}\left(Y_{\gamma}\right)$ are isomorphic for all $\gamma$ such that $S_{\gamma} \subset S_{\lambda}$.

Proof.

(1) All 2-cycles are conjugate in $\Sigma_{n}$ and every parabolic is generated by its $2-$ cycles.

(2) All the sign flips $t_{i}$ are conjugate. The $2-$ cycles and the elements of the form $t_{j} t_{k}(j, k)$ are all conjugate. Every parabolic is generated by its sign flips and $2-$ cycles.

(3) Again the 2-cycles and the elements of the form $t_{j} t_{k}(j, k)$ are all conjugate. Every parabolic is generated by such elements.

We now apply Corollary 34 to the periodic Toda lattices. The verifications of the various cases appear as discussion in Paper I, pages 140-146. The $G_{2}$ discussion is inadequate and partially incorrect as was mentioned in section 5 . We give a concise statement of these results in the lemma below. The proof for $G_{2}$ follows the statement.

Lemma 35. For the periodic Toda lattices with generic initial conditions, the images of the small loops around the ramification points under $\mu: \pi_{1}\left(P_{*}\right) \rightarrow W$ are as follows:

(1) If $\mathfrak{g}$ has type $A_{n-1}$ there are $2(n-1)$ loops $\beta_{i}$ with $\mu\left(\beta_{i}\right)$ conjugate to $(1,2)$ and 2 loops $\beta_{i}$ with $\mu\left(\beta_{i}\right)$ conjugate to an n-cycle.

(2) If $\mathfrak{g}$ has type $B_{n}$ or $C_{n}$ there are $2(n-1)$ loops $\beta_{i}$ with $\mu\left(\beta_{i}\right)$ conjugate to $(1,2)$, and 2 loops $\beta_{i}$ with $\mu\left(\beta_{i}\right)$ conjugate to $t_{1}$ and 2 loops $\beta_{i}$ with $\mu\left(\beta_{i}\right)$ conjugate to $t_{1}(1, \ldots, n)$.

(3) If $\mathfrak{g}$ has type $D_{n}$ there are $2 n$ loops $\beta_{i}$ with $\mu\left(\beta_{i}\right)$ conjugate to $(1,2)$ and 2 loops $\beta_{i}$ with $\mu\left(\beta_{i}\right)$ conjugate to $t_{1} t_{2}(2, \ldots, n)$.

(4) If $\mathfrak{g}$ has type $G_{2}$ there are 2 loops $\beta_{i}$ with $\mu\left(\beta_{i}\right)$ conjugate to $(1,2)$, and 2 loops $\beta_{i}$ with $\mu\left(\beta_{i}\right)$ conjugate to $t(1,2)$ and 2 loops $\beta_{i}$ with $\mu\left(\beta_{i}\right)$ conjugate to $t(1,2,3)$.

Note that $\mu$ is onto in each case.

Proof. For $G_{2}$ the representation with highest weight $\lambda_{1}$ is the restriction of the classical representation of $B_{3}$. For an appropriate choice of basis for the representation, the image of the Cartan is the set of matrices of the form

$$
\left(\begin{array}{ccccccc}
x_{1} & & & & & & \\
& x_{2} & & & & & \\
& & x_{3} & & & & \\
& & & 0 & & & \\
& & & & -x_{3} & & \\
& & & & & -x_{2} & \\
& & & & & & -x_{1}
\end{array}\right)
$$


with $x_{1}+x_{2}+x_{3}=0$. If we write this matrix as $\left[x_{1}, x_{2}, x_{3}\right]$ then the action of the Weyl group $\mathbf{Z}_{2} \times \Sigma_{3}$ is

$$
\left(t^{n}, \sigma\right)\left[x_{1}, x_{2}, x_{3}\right]=(-1)^{n+\operatorname{sign}(\sigma)}\left[x_{\sigma^{-1}(1)}, x_{\sigma^{-1}(2)}, x_{\sigma^{-1}(3)}\right] .
$$

Note that this is not the obvious action. The stabilizer of $\lambda_{1}$ is $S_{\lambda_{1}}=\langle t(2,3)\rangle$. The spectral curve $Y_{\lambda_{1}}$ for generic initial conditions is of the form $\{(s, z) \mid p(s, z)=$ $\left.z^{2}\left(z^{2}-c_{1}\right)^{2}+c_{0}+a\left(s+s^{-1}\right)=0\right\}$ where $s$ is the spectral parameter and $c_{0}, c_{1}$, and $a$ are constants depending on the initial conditions..

Label the points in the fiber over the basepoint in $P_{*}$ by their $z$ coordinates as $z_{1}, z_{2}, z_{3},-z_{1},-z_{2}$, and $-z_{3}$. They occur in $\pm z_{i}$ pairs since the polynomial is even in $z$. The fiber over the basepoint can be identified with the right cosets of $S_{\lambda_{1}}$. These cosets are given in a table in section 5. The spectral curve $Y_{\lambda_{1}}$ has one covering translation since the normalizer of $\langle t(2,3)\rangle$ is $\langle t\rangle$. The cover $Y_{\lambda_{1} *}$ maps equivariantly into $\left(G / H \times \mathfrak{h}_{*}\right) / S_{\lambda_{1}}$ by $(s, z) \rightarrow(g H, h)$. As a consequence from Paper I of Lemma 3 [MS], and Lemma 12 and its proof [MS] this map satisfies $A d_{g} h=A(s)$ and $z=\lambda_{1}(h)$. Since $t \cdot \lambda_{1}(h)=-\lambda_{1}(h)$, the covering translation is multiplication of the $z$ coordinate by -1 . So if $z_{i}$ is identified with $R_{\sigma}$ then $-z_{i}$ is identified with $t R_{\sigma}$. If $\beta$ is a small loop about a ramification point then the monodromy representation gives an action as permutations on the fiber by $R_{\sigma} \cdot \beta=R_{\sigma} \mu(\beta)$. Since $t$ is central, its monodromy action is the same as its action as a covering translation, i.e., multiplication by -1 . This fact and the table in section 5 of the $W$ action on the right cosets allow us to determine the conjugacy class of each $\mu(\beta)$ from its cycle decomposition.

Generically there are six ramification points, occurring in pairs with the same value of $\tau=a\left(s+s^{-1}\right)$. They occur at those values of $\tau$ where $p(s, z)$ and its partial derivative in $z$ have a common root. At $\tau=-c_{0}$, which is mislabelled as 0 in Paper $\mathrm{I}, p(s, z)=p(z)=z^{2}\left(z^{2}-c_{1}\right)^{2}$. Examination in a local coordinate shows that for $s=r e^{i \theta}$ as $\theta$ increases from 0 to $2 \pi$ three pairs of roots of $p(z)$ exchange and only one pair is a $\pm z_{i}$ pair. The table then gives that $\mu(\beta)$ is conjugate to $(1,2)$ for these two values of $s$. This particular $\mu(\beta)$ is given incorrectly in Paper I. A second pair of ramification points occur where $\tau=-c_{0}-(4 / 27) c_{1}^{3}$. At this value of $\tau$ we have $p(z)=(1 / 27)\left(3 z^{2}-c_{1}\right)^{2}\left(3 z^{2}-4 c_{1}\right)$. There are two pairs of roots exchanged by $\mu(\beta)$; hence it is conjugate to $t(1,2)$. The last value is at $\tau=\infty$. Changing to a local coordinate for $z$ reveals $\mu(\beta)$ to be a 6 -cycle and hence conjugate to $t(1,2,3)$. Note that any choice of representatives for these three conjugacy classes will generate $W$.

The immediate consequence of the above lemma is the following theorem.

Theorem 36. For the periodic Toda lattices for algebras of types $A, B, C, D$, or $G_{2}$ with generic initial conditions and for any fixed weight $\lambda$, the Prym-Tjurin varieties $\operatorname{Tur}_{\lambda}\left(Y_{\gamma}\right)$ are isomorphic for all $\gamma$ such that $S_{\gamma} \subset S_{\lambda}$. Furthermore the map $\pi_{\lambda \gamma}^{*}: \operatorname{Jac}\left(Y / S_{\lambda}\right) \rightarrow \operatorname{Jac}\left(Y / S_{\gamma}\right)$ is injective.

We now calculate the dimensions of the Prym-Tjurin varieties on which the flow for the periodic Toda lattices occur. For the periodic Toda lattices the parameter space is the Riemann sphere. The dimension of the Prym-Tjurin is given by the dimension formula from Lemma 16 and Theorem 17.

$$
\operatorname{dim} \operatorname{Tur}=\frac{1}{2}\left(\sum_{\text {ramification }}\left(\operatorname{dim} \mathfrak{h}-\operatorname{dim} \mathfrak{h}^{I_{x}}\right)-(\operatorname{dim} \mathfrak{h})(\text { Euler char }(P))\right)
$$


Suppose $\beta_{x}$ is a small loop about a ramification point $x$. By Lemma $18, I_{x}=$ $\left\langle\mu\left(\beta_{x}\right)\right\rangle$. In each case Lemma 35 is used to identify the number of ramification points and the conjugacy class of the associated stabilizer. The spectral curve $Y_{\lambda_{1}}$ agrees with the spectral curve of the representation of highest weight $\lambda_{1}$ from [AvM2]. This agreement follows from the fact that the weights of this representation form a single orbit.

type $A_{n}$. The fixed point sets are of dimension:

(1) $\operatorname{dim} \mathfrak{h}^{(1,2)}=n-2$,

(2) $\operatorname{dim} \mathfrak{h}^{(1, \ldots, n)}=0$

so $\operatorname{dim}$ Tur $=n-1$.

type $B_{n}$ or $C_{n}$. The fixed point sets are of dimension:

(1) $\operatorname{dim} \mathfrak{h}^{(1,2)}=n-1$,

(2) $\operatorname{dim} \mathfrak{h}^{t_{1}}=n-1$,

(3) $\operatorname{dim} \mathfrak{h}^{t_{1}(1, \ldots, n)}=0$,

so $\operatorname{dim}$ Tur $=n$. The flow takes place on the Prym variety from Theorem 25 which has dimension $n$, and fills this Prym [AvM2].

type $D_{n}$. The fixed point sets are of dimension:

(1) $\operatorname{dim} \mathfrak{h}^{(1,2)}=n-1$,

(2) $\operatorname{dim} \mathfrak{h}^{t_{1} t_{2}(1, \ldots, n)}=0$,

so $\operatorname{dim}$ Tur $=n$. Just as in the previous case the flow takes place on the Prym variety from Theorem 25 which has dimension $n$, and fills this Prym [AvM2].

type $G_{2}$. The fixed point sets are of dimension:

(1) $\operatorname{dim} \mathfrak{h}^{(1,2)}=1$,

(2) $\operatorname{dim} \mathfrak{h}^{t(1,2)}=1$,

(3) $\operatorname{dim} \mathfrak{h}^{t(1,2,3)}=0$,

so $\operatorname{dim}$ Tur $=2$. The genus of the spectral curve $Y_{\lambda_{1}}$ is 5 . The genus of the curve $Y_{A_{1}}$ (as described prior to Proposition 26) is 2 and the genus of $Y / \Sigma_{\mathrm{sk}}$ is 1 . The double cover $Y_{\lambda_{1}} \rightarrow Y_{A_{1}}$ defines a Prym variety of dimension 3 which properly contains $\operatorname{Tur}_{\lambda_{1}} Y_{\lambda_{1}}$. The Prym is isogenous to $\operatorname{Tur}_{\lambda_{1}} Y_{\lambda_{1}} \oplus \operatorname{Jac}\left(Y / \Sigma_{\text {sk }}\right)$ by Proposition 29. Adler and van Moerbeke showed that an isospectral set of initial conditions for the $G_{2}$ periodic Toda lattice maps onto a generic subset of a two-dimensional subtorus of the Prym [AvM2].

We conclude the paper with a discussion of the decomposition of the Jacobian of the $\operatorname{sl}(4, \mathbf{C})$ Toda master curve. There is some interest in splittable Jacobi varieties independently of integrable systems. See, for example, $[R]$ and its references. Here as an example we compute the multiplicities of the irreducible representations of the Weyl group $W=\Sigma_{4}$ that occur in the decomposition of the tangent space at the identity of the Jacobi variety of the master spectral curve for the periodic $\operatorname{sl}(4, \mathbf{C})$ Toda lattice with a generic initial condition. We go on to decompose the Jacobian itself into subabelian varieties. In general a $\mathbf{Z}[W]$ module, such as the period lattice used to give the Jacobian as a complex torus, need not decompose according to irreducible $\mathbf{C}[W]$ types. However, in this example each representation type in $T_{e} \operatorname{Jac} Y$ gives a subtorus of $\operatorname{Jac} Y$ and $\operatorname{Jac} Y$ is isogenous to the sum. Moreover, some of these subtori decompose further. The decomposition is obtained using induced representations and appears in Proposition 39. 
There are five irreducible representations of $\Sigma_{4}$ : the trivial representation, the alternating representation, the action on $\mathfrak{h}^{*}$, the corresponding action on $\bigwedge^{2} \mathfrak{h}^{*}$, and one more obtained as follows. There is an isomorphism $\Sigma_{4} \cong \mathbf{Z}_{2}^{2} \rtimes \Sigma_{3}$ from viewing $W=\Sigma_{4}$ as the Weyl group of type $D_{3}$ instead of type $A_{3}$. The group $\Sigma_{3}$ has a two-dimensional irreducible representation as symmetries of an equilateral triangle. The map $\mathbf{Z}_{2}^{2} \rtimes \Sigma_{3} \rightarrow \Sigma_{3}$ then gives a two-dimensional irreducible representation of $\Sigma_{4}$. These representations can be labelled by Young diagrams and matrices for them can be found in [Ha, p. 225].

To apply our multiplicity formula in the case of the periodic $\operatorname{sl}(4, \mathbf{C})$ Toda lattice, we need to to know the dimensions of the fixed point sets in these representations for a $2-$ cycle and a $4-$ cycle. The data appears in the following table.

\begin{tabular}{|c|c|c|c|c|c|}
\hline representation & trivial & alternating & $\mathfrak{h}^{*}$ & $\bigwedge^{2} \mathfrak{h}^{*}$ & triangle \\
\hline Young diagram & $\square$ & $\exists$ & $\mapsto \square$ & 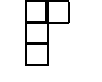 & $\square$ \\
\hline dim of rep & 1 & 1 & 3 & 3 & 2 \\
\hline dim fixed by $2-$ cycle & 1 & 0 & 2 & 1 & 1 \\
\hline dim fixed by $4-$ cycle & 1 & 0 & 0 & 1 & 1 \\
\hline Multiplicity in $T_{e} \mathrm{Jac} X$ & 0 & 3 & 3 & 5 & 2 \\
\hline dimension in $T_{e} \mathrm{Jac} X$ & 0 & 3 & 9 & 15 & 4 \\
\hline
\end{tabular}

The multiplicities of the irreducible representations of the Weyl group $W=\Sigma_{4}$ in the tangent space $T_{e} \mathrm{Jac} X$ at the identity of the Jacobi variety of the master curve $X$ are calculated by our formula which for $\chi$ nontrivial and self conjugate is

$$
\text { multiplicity of } \chi=\frac{1}{2}\left(\left(\sum_{\text {ramification }}\left(\operatorname{dim} \chi-\operatorname{dim} \chi^{I_{x}}\right)\right)-(\operatorname{dim} \chi)(\text { Euler char of } P)\right)
$$

and for the trivial representation is

$$
\text { multiplicity of } \chi_{\text {trivial }}=\frac{1}{2}(2-\text { Euler char of } P) \text {. }
$$

The dimensions in $T_{e}$ Jac $X$ are then the products of $\operatorname{dim} \chi$ and the respective multiplicity. The sum of the dimensions is 31 , the genus of the master spectral curve given in Paper I.

We now go on to decompose the $\operatorname{sl}(4, \mathbf{C})$ Toda lattice Jacobian of the master spectral curve. The symmetric group $\Sigma_{4}$ has 9 subgroups, up to conjugacy, besides itself and the trivial subgroup. They are:

$$
\begin{array}{ll}
A_{4} & \text { the alternating group, } \\
S_{8} & \text { the Sylow 2-subgroup which has order 8, } \\
\mathbf{Z}_{4}, \mathbf{Z}_{3}, \mathbf{Z}_{2} & \text { cyclic subgroups, } \\
\Sigma_{3}, \Sigma_{2}, \Sigma_{2} \times \Sigma_{2} & \text { parabolic subgroups, } \\
\mathbf{Z}_{2} \times \mathbf{Z}_{2} & \text { the subgroup }\{e,(12)(34),(13)(24),(14)(23)\} \subset A_{4} .
\end{array}
$$

The Sylow 2-subgroup has the structure $\mathbf{Z}_{4} \rtimes \mathbf{Z}_{2}$. One choice is to use $(1,2,3,4)$ to generate the $\mathbf{Z}_{4}$ summand and $(1,3)$ to generate the $\mathbf{Z}_{2}$ summand. Sylow's theorems show there is only one conjugacy class of Sylow 2-subgroups and there are 3 subgroups in this class. 
Induced representations can be decomposed using Frobenius reciprocity. Write -1 for the alternating representation and $\Delta$ for the representation labelled "triangle" above. As an example, we compute the multiplicity of $\Delta$ in $\operatorname{ind}_{S_{8}}^{\Sigma_{4}} 1$. By Frobenius reciprocity $\left\langle\operatorname{ind}_{S_{8}}^{\Sigma_{4}} 1, \Delta\right\rangle=\left\langle\operatorname{res}_{S_{8}}^{\Sigma_{4}} \Delta, 1\right\rangle$. Now $\Delta$ is the action of $\Sigma_{3}$ on a triangle via the map with kernel $\mathbf{Z}_{2} \times \mathbf{Z}_{2}$ :

$$
\{e\} \rightarrow \mathbf{Z}_{2} \times \mathbf{Z}_{2} \rightarrow \Sigma_{4} \rightarrow \Sigma_{3} \rightarrow\{e\} .
$$

Moreover $\mathbf{Z}_{2} \times \mathbf{Z}_{2} \subset S_{8}$, so the image of $S_{8}$ in $\Sigma_{3}$ is a 2-cycle. Consequently $S_{8}$ fixes one dimension of $\Delta$, so $\left\langle\operatorname{res}_{S_{8}}^{\Sigma_{4}} \Delta, 1\right\rangle=\operatorname{dim} \Delta^{S_{8}}=1$.

We will use the following decompositions.

$$
\begin{aligned}
& \operatorname{ind}_{A_{4}}^{\Sigma_{4}} 1=1 \oplus-1, \\
& \operatorname{ind}_{S_{8}}^{\Sigma_{4}} 1=1 \oplus \Delta, \\
& \operatorname{ind}_{\mathbf{Z}_{4}}^{\Sigma_{4}} 1=1 \oplus \Delta \oplus \bigwedge \mathfrak{h}, \\
& \operatorname{ind}_{\Sigma_{3}}^{\Sigma_{4}} 1=1 \oplus \mathfrak{h} .
\end{aligned}
$$

We now bring Lemma 28 to bear. First off, the parameter space is the Riemann sphere. From Lemma 28(4) the component of $T_{e}(\mathrm{Jac} Y)$ coming from the trivial representation is $d \pi^{*}\left(T_{e} P\right)$. Hence there is no trivial component in $T_{e}(\mathrm{Jac} Y)$ or for any of the quotient curves.

Lemma 28(1) gives $T_{e}\left(\mathrm{Jac} Y / A_{4}\right)=\left(T_{e} \mathrm{Jac} Y / A_{4}\right)_{-1}$. Since the restriction of the alternating representation to the alternating group is trivial, $\left(T_{e} \operatorname{Jac} Y\right)_{-1}=$ $d \pi_{A_{4}}^{*}\left(T_{e} \mathrm{Jac} Y / A_{4}\right)$ which exponentiates to the torus $\pi_{A_{4}}^{*}\left(\mathrm{Jac} Y / A_{4}\right)$.

Again Lemma 28(1) gives $T_{e}\left(\operatorname{Jac} Y / \Sigma_{3}\right)=\left(T_{e} \operatorname{Jac} Y / \Sigma_{3}\right)_{\mathfrak{h}}$. Lemma 28(3) tells us that $\operatorname{dim}\left(T_{e} \operatorname{Jac} Y / \Sigma_{3}\right)_{\mathfrak{h}}=3 \operatorname{dim} \mathfrak{h}^{\Sigma_{3}}=3$. This is not all of $\left(T_{e} \operatorname{Jac} Y / \Sigma_{3}\right)_{\mathfrak{h}}$ which has dimension 9 , but this component of the tangent space is a direct sum of images $d \pi_{\Sigma_{i j k}}^{*}\left(T_{e} \operatorname{Jac} Y / \Sigma_{i j k}\right)$ for $\Sigma_{i j k}$ ranging over any three of the 4 subgroups conjugate to $\Sigma_{3}$. We use the natural names $\Sigma_{123}, \Sigma_{124}, \Sigma_{134}$, and $\Sigma_{234}=\Sigma_{3}$ for these subgroups. We need some lemmas.

Lemma 37. If $\operatorname{ind}_{S}^{W} 1=1 \oplus \chi$ for an irreducible representation $\chi$ of $W$ and if $w S w^{-1} \neq S$, then $\chi^{S} \cap \chi^{w S w^{-1}}=\{0\}$ and consequently $\pi_{S}^{*}(\operatorname{Jac} Y / S)_{\chi}$ is tranverse to $\pi_{w S w^{-1}}^{*}\left(\operatorname{Jac} Y /\left(w S w^{-1}\right)\right)_{\chi}$.

Proof. First we show there is no subgroup $T$ with proper inclusions $S \subset T \subset W$. If there were such a subgroup $T$ then

$$
\begin{aligned}
\operatorname{ind}_{S}^{W} 1 & =\operatorname{ind}_{T}^{W}\left(\operatorname{ind}_{S}^{T} 1\right) \\
& =\operatorname{ind}_{T}^{W}(1 \oplus \gamma)
\end{aligned}
$$

for some representation $\gamma$, not necessarily irreducible, of $T$

$$
\begin{aligned}
& =\operatorname{ind}_{T}^{W} 1 \oplus \operatorname{ind}_{T}^{W} \gamma \\
& =\left(1 \oplus \gamma^{\prime}\right) \oplus \operatorname{ind}_{T}^{W} \gamma
\end{aligned}
$$

where $\gamma^{\prime}$ is a representation of $W$. So in this case ind ${ }_{S}^{W} 1$ has at least 3 irreducible factors rather than just 2 . So there is no such subgroup $T$.

If $S \neq w S w^{-1}$ then they generate $W$. So $\chi^{S} \cap \chi^{w S w^{-1}}$ is fixed by $\left\langle S, w S w^{-1}\right\rangle=$ $W$ and if nonzero would give a trivial representation of $W$ inside $\chi$. Hence $\chi^{S} \cap$ 
$\chi^{w S w^{-1}}=\{0\}$. The transversality of $\pi_{S}^{*}(\operatorname{Jac} Y / S)_{\chi}$ and $\pi_{w S w^{-1}}^{*}\left(\operatorname{Jac} Y /\left(w S w^{-1}\right)\right)_{\chi}$ then follows via a transfer argument.

Lemma 38. If $\operatorname{dim} \chi^{S}=1$ then there is a subset $\mathcal{S} \subset W$ such that

$$
(\operatorname{Jac} Y)_{\chi}=\underset{w \in \mathcal{S}}{\oplus} \pi_{w S w^{-1}}^{*}\left(\operatorname{Jac} Y /\left(w S w^{-1}\right)\right)_{\chi}
$$

up to isogeny.

Proof. We check the decomposition on the level of tangent spaces. By a transfer argument

$$
d \pi_{w S w^{-1}}^{*}\left(T_{e} \operatorname{Jac} Y /\left(w S w^{-1}\right)\right)_{\chi}=\left(T_{e} \operatorname{Jac} Y\right)_{\chi}^{w S w^{-1}} .
$$

Now $\left(T_{e} \mathrm{Jac} Y\right)_{\chi}$ is a direct sum of copies of $\chi$. Due to the $W$ invariance of each copy of $\chi$ it is adequate to look at a single copy. By assumption $\operatorname{dim} \chi^{S}=1$, so $S$ fixes the span of a nonzero vector $v$. Since $\chi$ is irreducible, its representation space is spanned by the orbit $W \cdot v$, or equivalently, by the fixed point sets of groups in the conjugacy class $\left\{w S w^{-1} \mid w \in W\right\}$. Pick a basis for the representation space contained in the orbit $W \cdot v$ and let $\mathcal{S}=\{w\}$ such that $\{w \cdot v\}$ is the chosen basis. Then $\chi=\underset{w \in \mathcal{S}}{\oplus} \chi^{w S w^{-1}}$ and so $\left(T_{e} \operatorname{Jac} Y\right)_{\chi}=\underset{w \in \mathcal{S}}{\oplus} d \pi_{w S w^{-1}}^{*}\left(T_{e} \operatorname{Jac} Y /\left(w S w^{-1}\right)\right)_{\chi}$.

If, as usual, we view $\Sigma_{3} \subset \Sigma_{4}$ as the permutations that fix the first element, and $\mathfrak{h} \subset \mathbf{C}^{4}$ as the orthogonal complement of the vector $(1,1,1,1)$, then $\mathfrak{h}^{\Sigma_{3}}$ is the onedimensional subspace spanned by $v=(-3,1,1,1)$. In fact $\mathfrak{h}=\mathfrak{h}^{\Sigma_{234}} \oplus \mathfrak{h}^{\Sigma_{123}} \oplus \mathfrak{h}^{\Sigma_{124}}$. The curves $Y / \Sigma_{i j k}, 1 \leq i \leq j \leq k \leq 4$, are all isomorphic. So we may write the isogeny

$$
(\operatorname{Jac} Y)_{\mathfrak{h}}=3\left(\operatorname{Jac} Y / \Sigma_{3}\right)
$$

The situation for $(\operatorname{Jac} Y)_{\Delta}$ is similar. Jac $Y / S_{8}=\left(\operatorname{Jac} Y / S_{8}\right)_{\Delta}$. As discussed previously $\Delta^{S_{8}}$ is one dimensional while $\Delta$ is 2 dimensional. We get the isogeny

$$
(\mathrm{Jac} Y)_{\Delta}=2\left(\operatorname{Jac} Y / S_{8}\right) .
$$

The last component $(\operatorname{Jac} Y)_{\Lambda^{2} \mathfrak{h}}$ is isogenous to a sum of 3 copies of a Prym variety. Consider the double cover $\pi: Y / \mathbf{Z}_{4} \rightarrow Y / S_{8}$. Since $\operatorname{dim} \Delta^{\mathbf{Z}_{4}}=1=$ $\operatorname{dim} \Delta^{S_{8}}$, by Lemma $28(4)$ the map $d \pi^{*}: T_{e}\left(\operatorname{Jac} Y / S_{8}\right) \rightarrow\left(T_{e}\left(\operatorname{Jac} Y / \mathbf{Z}_{4}\right)\right)_{\Delta}$ is onto.

We now explain why $\left(T_{e} \mathrm{Jac} Y / \mathbf{Z}_{4}\right)_{\wedge^{2} \mathfrak{h}}$ is the tangent space at the identity of the Prym variety of the double cover $\pi$. The tangent space of the Prym variety can be found as either the image of $d(1-\iota)$ where $\iota$ is the involution that interchanges the sheets of the double cover, or, more conveniently, the kernel of $d \pi$. Now $d \pi$ is an isomorphism from $\left(T_{e} \mathrm{Jac} Y / \mathbf{Z}_{4}\right)_{\Delta}$ to $T_{e} \mathrm{Jac} Y / S_{8}$ and is zero on $\left(T_{e} \mathrm{Jac} Y / \mathbf{Z}_{4}\right)_{\bigwedge^{2} \mathfrak{h}}$ since there is no $\bigwedge^{2} \mathfrak{h}$ summand in $T_{e} \mathrm{Jac} Y / S_{8}$. Since $T_{e} \operatorname{Jac} Y / \mathbf{Z}_{4}=\left(T_{e} \operatorname{Jac} Y / \mathbf{Z}_{4}\right)_{\Delta} \oplus\left(T_{e} \operatorname{Jac} Y / \mathbf{Z}_{4}\right)_{\bigwedge^{2} \mathfrak{h}}$, the $\bigwedge^{2} \mathfrak{h}$ summand must be the kernel.

The image of the tangent space to the Prym variety,

$$
d \pi_{\mathbf{Z}_{4}}\left(T_{e}\left(\operatorname{Prym}\left(Y / \mathbf{Z}_{4} \rightarrow y / S_{8}\right)\right)\right)
$$

in $T_{e}$ Jac $Y$ has one dimension for each copy of $\bigwedge^{2} \mathfrak{h}$ in $T_{e}$ Jac $Y$ since $\mathbf{Z}_{4}$ fixes a one-dimensional subspace of $\Lambda^{2} \mathfrak{h}$. In fact the action of the $W=\Sigma_{4}$ on $\Lambda^{2} \mathfrak{h}$ is isomorphic to the skewed permutation action on the subspace of $\mathbf{C}^{4}$ orthogonal to $(1,1,1,1)$ in which $\sigma \in W$ acts by permuting the entries and multiplication by the determinant of the permutation. As in the earlier cases $W$ contains three 
conjugate subgroups that are cyclic of order four and these contribute three copies of the Prym variety to make up the $\bigwedge^{2} \mathfrak{h}$ summand of Jac $Y$. We summarize.

Proposition 39. The Jacobian of the master spectral curve for the $\operatorname{sl}(4, \mathbf{C})$ Toda lattice with generic initial conditions decomposes up to isogeny as

$$
\operatorname{Jac} Y=\operatorname{Jac} Y / A_{4} \oplus 3 \operatorname{Jac} Y / \Sigma_{3} \oplus 2 \operatorname{Jac} Y / S_{8} \oplus 3 \operatorname{Prym}\left(Y / \mathbf{Z}_{4} \rightarrow Y / S_{8}\right) .
$$

\section{REFERENCES}

[A] Robert D.M. Accola, Vanishing Properties of Theta Functions for Abelian Covers of Riemann Surfaces, Advances in the Theory of Riemann Surfaces, Proceedings of the 1969 Stony Brook Conference, Princeton University Press, Princeton, N.J., 1971, pp. 718. MR 44:5448

[AvM1] M. Adler and P. van Moerbeke, Completely Integrable Systems, Euclidean Lie Algebras, and Curves, Advances in Math. 38 (1980), 267-317. MR 83m:58041

[AvM2] _ Linearization of Hamiltonian Systems, Jacobi Varieties, and Representation Theory, Advances in Math. 38 (1980), 318-379. MR 83m:58042

[BE] Robert J. Baston and Michael G. Eastwood, The Penrose Transform: Its Interaction with Representation Theory, Oxford University Press, New York, N.Y., 1989. MR 92j:32112

[BL] Ch. Birkenhake and H. Lange, Norm-endomorphisms of abelian subvarieties, Classification of Irregular Varieties, Lecture Notes in Mathematics, vol. 1515, Springer-Verlag, Berlin, 1992, pp. 21-32. MR 93g:14051

[B] Armand Borel, et al., Seminar on Transformation Groups, Annals of Mathematics Studies, Number 46, Princeton University Press, Princeton, N.J., 1960. MR 22:7129

[BMP] M.R. Bremner, R.V. Moody, and J. Patera, Tables of Dominant Weight Multiplicities for Representations of Simple Lie Algebras, Pure and Applied Mathematics, vol. 90, Marcel Dekker, Inc., New York, N.Y., 1985. MR 86f:17002

[BD] Theodor Bröcker and Tammo tom Dieck, Representations of Compact Lie Groups, GTM, vol. 98, Springer-Verlag, New York, N.Y., 1985. MR 86i:22023

[CR] Charles W. Curtis and Irving Reiner, Methods of Representation Theory, vol. I, John Wiley \& Sons, New York, N.Y., 1981. MR 82i:20001

[G] Phillip A. Griffiths, Linearizing Flows and a Cohomological Interpretation of Lax Equations, American Journal of Mathematics 107 (1985), 1445-1483. MR 87e:58048

[GH] Phillip A. Griffiths and Joseph Harris, Principles of Algebraic Geometry, John Wiley \& Sons, New York, N.Y., 1978. MR 80b:14001

[Ha] Morton Hamermesh, Group Theory and its Application to Physical Problems, Dover Publications, Inc., New York, N.Y., 1962. MR 25:132

[H1] James E. Humphreys, Reflection Groups and Coxeter Groups, Cambridge University Press, Cambridge, Great Britain, 1990.

[H2] Introduction to Lie algebras and representation theory, GTM, vol. 9, SpringerVerlag, New York, N.Y., 1972. MR 48:2197

[K] Vassil I. Kanev, Spectral Curves, Simple Lie Algebras, and Prym-Tjurin Varieties, Proceedings of Symposia in Pure Mathematics 49 (1989), 627-645. MR 91b:14028

[Kr] Aloys Krieg, Hecke Algebras, Memoirs of the American Mathematical Society 87 (435) (1990). MR 90m:16024

[M] William Massey, Algebraic Topology: An Introduction, Harcourt-Brace, New York, N.Y., 1967. MR 35:2271

[Mc1] Andrew McDaniel, Lie Algebra Representations and the Toda lattice, thesis, Brandeis University (1985).

[Mc2] A. McDaniel, Representations of $\operatorname{sl}(n, \mathbf{C})$ and the Toda lattice, Duke Math. Journal 56 (1988), 47-99. MR 89e:58061

[MS] A. McDaniel and L. Smolinsky, A Lie Theoretic Galois Theory for the Spectral Curves of an Integrable System: I, Communications in Mathematical Physics 149 (1992), 127-148. MR 94f: 58065

[MS1] A. McDaniel and L. Smolinsky, The Flow of the $G_{2}$ Periodic Toda Lattice, preprint.

[OP] M.A. Olshanetsky and A.M. Perelomov, Classical Integrable Systems Related to Lie Algebras, Physics Reports 71 (1981), 313-400. MR 83d:58032 
[P] Stefanos Pantazis, Prym Varieties and the Geodesic Flow on SO(n), Mathematische Annalen 273 (1986), 297-315. MR 87g:58054

[R] John F. X. Ries, Splittable Jacobi Varieties, Curves, Jacobians, and Abelian Varieties, Contemporary Mathematics, vol. 136, American Mathematical Society, Providence, R.I., 1992, pp. 305-326. MR 93j:14031

[vMM] P. van Moerbeke and D. Mumford, The Spectrum of Difference Operators and Algebraic Curves, Acta Math. 143 (1979), 93-154. MR 80e:58028

Department of Mathematics, Georgetown University, Washington, D.C. 20057

E-mail address: Mcdaniea@guvax.georgetown.edu

Department of Mathematics, Louisiana State University, Baton Rouge, Louisiana 70803

E-mail address: smolinsk@math.lsu.edu 